\title{
Stability of de Sitter spacetime under isotropic perturbations in semiclassical gravity
}

\author{
Guillem Pérez-Nadal \\ Departament de Física Fonamental, Universitat de Barcelona, Avinguda Diagonal 647, 08028 Barcelona, Spain
}

Albert Roura

Theoretical Division, T-8, Los Alamos National Laboratory, Mail Stop B285, Los Alamos, New Mexico 87545, USA

Enric Verdaguer

Departament de Física Fonamental and Institut de Ciències del Cosmos, Universitat de Barcelona, Avinguda Diagonal 647, 08028 Barcelona, Spain

(Received 14 December 2007; published 23 June 2008)

\begin{abstract}
A spatially flat Robertson-Walker spacetime driven by a cosmological constant is nonconformally coupled to a massless scalar field. The equations of semiclassical gravity are explicitly solved for this case, and a self-consistent de Sitter solution associated with the Bunch-Davies vacuum state is found (the effect of the quantum field is to shift slightly the effective cosmological constant). Furthermore, it is shown that the corrected de Sitter spacetime is stable under spatially isotropic perturbations of the metric and the quantum state. These results are independent of the free renormalization parameters.
\end{abstract}

DOI: 10.1103/PhysRevD.77.124033

PACS numbers: 04.62.+v, 98.80.- $\mathrm{k}$

\section{INTRODUCTION}

Our present understanding of cosmology assumes that the Universe underwent a short period of accelerated expansion known as inflation [1-5]. The inflationary scenario has been remarkably successful in explaining the observed anisotropies of the cosmic microwave background [6-9]. In most inflationary models the accelerated expansion phase is close to but never exactly de Sitter and this phase eventually ends when the kinetic energy of the inflaton field driving inflation starts to dominate over the potential term. On the other hand, observations of distant supernovae indicate that the Universe is presently undergoing a period of accelerated expansion $[10,11]$ that may be driven by a small nonvanishing cosmological constant [12-15]. If that is the case, the geometry of our Universe would tend to that of de Sitter spacetime at sufficiently late times. Thus, a detailed knowledge of the physics associated with de Sitter space may play a key role in understanding both the very early Universe as well as its ultimate fate. Furthermore, it is conceivable that studying a possible screening of the cosmological constant driving de Sitter space, due to quantum effects, could shed some light on the huge fine-tuning problem that the current value of the cosmological constant seems to pose.

An open question which has recently received increasing attention is whether the quantum fluctuations of the metric and the matter fields in de Sitter space can give rise to large backreaction effects on the mean background geometry. It has been argued that in pure gravity with a cosmological constant the infrared effects due to two graviton loops and higher-order radiative corrections could lead to a secular screening of the cosmological constant $[16,17]$. There have also been proposals that a significant screening of the cosmological constant could appear in chaotic inflationary models at one loop when both the metric and the inflaton field fluctuations are considered [18-21]. In all these cases the quantum fluctuations of the metric play an essential role. However, whenever the metric perturbations are quantized, one needs to confront the problem of defining proper diffeomorphism-invariant observables in quantum gravity [22], even when treated as a low-energy effective field theory. In particular one needs to make sure that the secular screening found in the analysis mentioned above is not simply a gauge artifact. As a matter of fact, it was shown in Refs. $[23,24]$ that when a suitable gauge-invariant measure of the expansion rate was considered the screening effect previously found in chaotic inflationary models was not actually present (at least for single field models). Similarly, a recent reanalysis of the pure gravity case which made use of a diffeomorphism-invariant measure of the change of the expansion rate revealed the absence of secular effects to all orders in perturbation theory [25] (although this conclusion is still subject to certain debate [26]).

In recent work it was also found that the backreaction due to one-loop effects of massless nonconformal fields can give rise to substantial deviations from de Sitter spacetime [27]. This would be the case even if the quantum fluctuations of the metric are not considered (provided that there is some other massless nonconformal field in addition to gravitons). Not quantizing the metric perturbations means that the ambiguity associated with the gauge-fixing term for the metric perturbations is no longer present, and the mean geometry is a perfectly well-defined (and gaugeindependent) object. Hence, the subtleties mentioned in the previous paragraph do not apply. The heuristic argument provided in Ref. [27] is that one-loop contributions from 
massless nonconformal fields correspond to logarithmic nonlocal terms [conformal fields only produce local terms for Robertson-Walker (RW) geometries], and by analogy with the situation in pion physics, one expects that they become important in the infrared limit. However, it is not clear that pion physics constitutes a good analogy because the derivative coupling of the matter fields to the metric generates higher powers of the momentum. This point can be illustrated with the simple example of small metric perturbations around flat space. In that case the Fourier transform of the inverse propagator behaves like $k^{2}[1+$ $\left.b\left(k^{2} / m_{p}^{2}\right) \ln \left(k^{2} / \mu_{0}^{2}\right)\right]$, where $b$ is a dimensionless number roughly of order $1, m_{p}$ is the Planck mass and $\mu_{0}$ is some fixed mass scale. One can see that although the logarithm grows in the infrared, the whole term actually decreases because it is suppressed by the factor $\left(k^{2} / m_{p}^{2}\right)$. Indeed, a detailed calculation of the quantum radiative corrections to the Newtonian potential shows that the contribution from that term is suppressed by the square of the ratio of the Planck length over the radial distance [28-30]. Of course the case of a RW metric, which involves a time-dependent scale factor, is not so simple and deserves a careful analysis in order to compare with the detailed calculation in Ref. [27].

In this paper we approach this problem by explicitly solving the backreaction on the mean gravitational field due to the quantum effects of a massless nonconformally coupled scalar field when the quantum fluctuations of the metric are not considered. This kind of one-loop calculation is entirely equivalent to studying the corresponding backreaction problem in the semiclassical gravity framework [31-33] by solving self-consistently the semiclassical Einstein equation, which includes the suitably renormalized quantum expectation value of the stress-tensor operator acting as a source. Specifically, in our calculation we assume the presence of a cosmological constant, which would lead to a de Sitter solution in the absence of quantum effects, and simplify the problem by focusing on RW geometries, corresponding to spatially homogenous and isotropic states of the quantum field. It should be emphasized that a complete analysis of the backreaction problem in de Sitter spacetime and its stability should include the effect of the quantum metric fluctuations as well. However, as mentioned above, including the quantum fluctuations of the metric in a satisfactory way is technically rather involved and entails a number of conceptual subtleties. It is, therefore, important to make sure first that there are no significant effects even when the quantum metric fluctuations are not taken into account, especially because the existence of such effects has actually been suggested by a number of studies in the literature.

There exist relevant antecedents to our analysis in the context of quantum field theory in a fixed curved spacetime, i.e., when the backreaction of the quantum fields on the spacetime geometry is not taken into account. The so- called Bunch-Davies vacuum [31,34] for fields in de Sitter is a state invariant under all the isometries of de Sitter space, which is maximally symmetric. The renormalized expectation value of the stress-tensor operator for that state is proportional (with a constant factor) to the metric and therefore its contribution to the semiclassical Einstein equation has the same form as a cosmological constant term. As is well known, this makes it possible to have selfconsistent de Sitter solutions of the semiclassical equation [35-37] (this has also been found in models with modified dispersion relations for trans-Planckian frequencies [38]). More importantly, it was shown in Ref. [39] that for fields with a wide range of mass and curvature-coupling parameters evolving in a given de Sitter spacetime, the expectation value of the stress tensor for any reasonable initial state tended at late times to the expectation value for the BunchDavies vacuum, where by reasonable states one means states with the same ultraviolet behavior as the Minkowski vacuum, i.e., with essentially no excitations at arbitrarily high frequencies (technically they are known as fourth-order adiabatic states $[31,40])$. This result can be intuitively understood as follows: the exponential expansion will redshift any finite frequency excitations of the Bunch-Davies vacuum so that their contribution to the stress tensor will tend to zero at late times.

The result described in the previous paragraph suggests that even when taking into account backreaction effects, perturbations around de Sitter will be redshifted away and at late times the spacetime geometry will approach de Sitter space with an effective cosmological constant which includes the contribution from the expectation value of the stress tensor for the Bunch-Davies vacuum. However, in order to prove this expectation without any room for doubt, one needs to solve both the semiclassical Einstein equation and the Klein-Gordon equation for the scalar field self-consistently. This is the main goal of this paper. We will consider a fairly general family of Gaussian initial states for the quantum field which are spatially homogenous and isotropic, and discuss under what conditions the trace of the stress-tensor expectation value exhibits unphysical divergences at the initial time after the standard renormalization procedure. We will also explain how to select appropriate states with regular initial behavior, and then solve the backreaction equation explicitly. We obtain relatively simple analytic expressions for the solutions at all times. The standard renormalization procedure for the ultraviolet divergences of the expectation value of the stress tensor requires the renormalization of the gravitational and cosmological constants as well as two new dimensionless parameters which are related to local geometric terms in the gravitational action which are quadratic in the curvature tensor. These new parameters should in principle be determined experimentally in order to eliminate the two-parameter ambiguity otherwise exhibited by the backreaction equation. Nevertheless, for the 
particular case that we are considering (and at the order in the Planck length at which we are working) the results turn out to be independent of the particular value of these renormalization parameters.

The plan of the paper is the following. In Sec. II we introduce the particular model that we will be considering and describe the procedure that we will employ to generate the initial state of the quantum field. In Sec. III we use the closed time path (CTP) formalism, whose application to semiclassical gravity is briefly summarized in the Appendix, to derive the effective action which encodes the effect of the quantum fields on a spatially flat RW spacetime. We also derive the relation between the timetime component of the stress tensor and its trace, which will be later used to derive the backreaction equation. In Sec. IV the CTP effective action is used to obtain the quantum expectation value of the stress-tensor operator, and the suitable initial conditions for the quantum state of the field and for the cosmological scale factor are discussed in detail. Finally, in Sec. V the semiclassical Friedmann equation that describes the backreaction of the quantum field on the scale factor, driven by a cosmological constant, is derived and solved perturbatively in powers of the Planck length over the Hubble radius. We find an explicit result valid for all times. It shows that there exists a selfconsistent de Sitter solution with a slightly shifted cosmological constant due to the one-loop effects and that all the other solutions tend to this one at late times. The main results are summarized in Sec. VI, where natural generalizations of the work presented here are also discussed. Some aspects of the functional approach to semiclassical gravity and the renormalization of the stress-tensor expectation value used in the paper are summarized in Appendix A. Finally, the connection of the initial states that we consider with the fourth-order adiabatic states and the method of adiabatic regularization in RW spacetimes is explained in Appendix B. Throughout the paper we use natural units with $\hbar=c=1$ and the $(+,+,+)$ convention of Ref. [41].

\section{A QUANTUM FIELD IN A RW BACKGROUND AND ITS INITIAL STATE}

In this section we describe our model for the backreaction of quantum fields on a cosmological background. We assume a spatially homogeneous and isotropic cosmological model with flat spatial sections, described by the metric

$$
g_{\mu \nu}=a^{2}(\eta) \eta_{\mu \nu}
$$

where $\eta_{\mu \nu}$ is the $n$-dimensional Minkowski metric (we use arbitrary dimensions for the moment in order to perform dimensional regularization later on), which takes the form $\eta_{\mu \nu}=\operatorname{diag}(-1,1, \ldots, 1)$ when considering the usual inertial coordinates, and $a(\eta)$ is the cosmological scale factor in terms of the conformal time $\eta$, which is related to the physical time $t$ by $a d \eta=d t$. The classical action for a real massless scalar field $\Phi(x)$ coupled to gravity is

$$
\begin{aligned}
S_{m}\left[g_{\mu \nu}, \Phi\right]= & -\frac{1}{2} \int d^{n} x \sqrt{-g}\left[g^{\mu \nu} \partial_{\mu} \Phi \partial_{\nu} \Phi\right. \\
& \left.+\left(\xi_{c}+\nu\right) R \Phi^{2}\right],
\end{aligned}
$$

where the dimensionless parameters $\xi_{c} \equiv(n-2) /[4(n-$ 1)] (equal to $1 / 6$ in four dimensions) and $\nu$ give the coupling to the Ricci curvature scalar $R$, given in this case by

$$
R=2(n-1)\left(\frac{\ddot{a}}{a^{3}}+\frac{n-4}{2} \frac{\dot{a}^{2}}{a^{4}}\right),
$$

where here and throughout the rest of the paper overdots denote derivatives with respect to the conformal time, i.e., $\cdot \equiv d / d \eta$. The minimal coupling case (no direct coupling to the curvature) corresponds to $\nu=-\xi_{c}$; a massless scalar field with minimal coupling mimics the behavior of gravitons in the cosmological background, except for a factor of 2 corresponding to the graviton polarizations. When $\nu=0$ the classical action $S_{m}\left[g_{\mu \nu}, \Phi\right]$ is invariant under conformal transformations with $g_{\mu \nu} \rightarrow \Omega^{2}(x) g_{\mu \nu}$ and $\Phi(x) \rightarrow \Omega^{(2-n) / 2}(x) \Phi(x)$; this is known as the conformal coupling case and it can be used to mimic the behavior of photons.

Since the RW metric given by Eq. (1) is conformally flat, it is convenient to introduce the rescaled scalar field $\phi(x)=a^{(n-2) / 2}(\eta) \Phi(x)$. The action in Eq. (2) then simplifies to

$$
S_{m}[a, \phi]=-\frac{1}{2} \int d^{n} x\left(\eta^{\mu \nu} \partial_{\mu} \phi \partial_{\nu} \phi+\nu a^{2} R \phi^{2}\right),
$$

which is the action for a free scalar field $\phi(x)$ in Minkowski spacetime with a time-dependent quadratic coupling $\nu a^{2} R \phi^{2}$. Identifying the matter Lagrangian $L_{m}$ from $S_{m}=\int d \eta L_{m}$ and the momentum $\pi(x)=$ $\delta L_{m} / \delta \dot{\phi}(x)=\dot{\phi}(x)$, one obtains the following Hamiltonian for the rescaled scalar field:

$$
\begin{aligned}
H_{m}[\pi, \phi] & =\frac{1}{2} \int d^{n-1} x\left[\pi^{2}+(\vec{\nabla} \phi)^{2}+\nu a^{2} R \phi^{2}\right] \\
& \equiv H_{m}^{(0)}+H_{\mathrm{int}},
\end{aligned}
$$

where in the second equality we have separated the Hamiltonian into that of a free massless field, $H_{m}^{(0)}$, and an interaction Hamiltonian $H_{\text {int }}$, which is proportional to $\nu$.

Let us now discuss the kind of initial quantum states of the field that we will be considering. We are interested in the evolution of the scale factor driven by a cosmological constant $\Lambda$ plus the backreaction effect due to the quantum scalar field, given some initial conditions for the scale factor and its derivative at some initial time $\eta_{i}$ as well as the initial state of the quantum field at that time. On the other hand, we will use the evolution from $\eta=-\infty$ to $\eta=\eta_{i}$ as an auxiliary way to prepare the initial quantum 
state of the field. More precisely, the initial state of the field will be given by

$$
\left|\Psi_{i}\right\rangle=\hat{U}\left(\eta_{i},-\infty\right)|0,-\infty\rangle
$$

where $|0,-\infty\rangle$ is the usual Minkowski vacuum at $\eta \rightarrow$ $-\infty$ and $\hat{U}$ is the time evolution operator associated with the Hamiltonian given by Eq. (5). $\hat{U}\left(\eta_{i},-\infty\right)$ only depends on the scale factor before the initial time $\eta_{i}$, which will be denoted from now on by $a_{\Psi}(\eta)$ and can be a fairly arbitrary regular function, subject only to the condition

$$
\lim _{\eta \rightarrow-\infty} a_{\Psi}^{2}(\eta) R_{\Psi}(\eta)=0
$$

where $R_{\Psi}(\eta)$ is the Ricci scalar corresponding to $a_{\Psi}(\eta)$, and to the requirement of a sufficiently smooth transition at $\eta_{i}$ to the scale factor at later times (the reason for this latter condition will be explained in detail in Sec. IV). Thus, our initial state is a squeezed state ${ }^{1}$ that evolves (in the Schrödinger picture) from the Minkowski vacuum state. A particular case is the Bunch-Davies vacuum [34] for zero mass and curvature coupling $\left(\xi_{c}+\nu\right)$, which would follow from considering a scale factor $a_{\Psi}(\eta)$ that corresponds to a given de Sitter spacetime all the way from $\eta=-\infty$ to $\eta=\eta_{i}$ [note that such a scale factor does satisfy condition (7)]. Note that the state obtained from the construction described above and defined by Eq. (6) is the state of the rescaled field $\phi(x)$. However, the state of the original field $\Phi(x)$ can be derived straightforwardly from it if one takes into account the simple relation between $\phi(x)$ and $\Phi(x)$ involving the scale factor $a(\eta)$.

Finally, the gravitational action for the scale factor can be written as

$$
S_{g}[a]=\frac{\mathcal{V}}{2 \kappa} \int_{\eta_{i}} d \eta a^{n}(R-2 \Lambda)+S_{g}^{c}[a]
$$

where the first term is just the Einstein-Hilbert term with $\kappa=8 \pi G=8 \pi / m_{p}^{2}(G$ is the gravitational coupling constant, $m_{p}$ is the Planck mass and we are using natural units with $\hbar=c=1), \mathcal{V}=\int d^{n-1} x$ is a spatial comoving volume factor that will drop in the final expressions, and $S_{g}^{c}[a]$ accounts for the gravitational counterterms that will be specified later. At this point the parameters $\kappa$ and $\Lambda$ should in principle be considered as bare parameters. However, for a massless field these parameters do not need to be renormalized when using dimensional regularization since the divergences in that case only require counterterms which are quadratic in the curvature, as we will see below.

\footnotetext{
${ }^{1}$ This means that we restrict our attention to a family of Gaussian pure states.
}

\section{THE EFFECTIVE ACTION FOR THE COSMOLOGICAL SCALE FACTOR}

\section{A. The expectation value of the energy density and the trace of the stress tensor}

We can now follow the procedure outlined in Appendix A to derive the semiclassical Einstein equation describing the backreaction of the scalar field on the spacetime geometry. The expectation value of the stress tensor for a given state of the field plays a key role in that equation. As long as one considers spatially homogenous and isotropic states of the quantum fields, it is consistent to assume that the metric in the semiclassical equation (A1) takes the restricted form (1) throughout, so that there is only one dynamical variable $a(\eta)$ to be determined.

Hence, we can concentrate on just one of the equations for the different components of Eq. (A1), and, in particular, on the 00 component, which in this semiclassical cosmological context may be called the semiclassical Friedmann equation. The expectation value $\left\langle\hat{T}_{00}\right\rangle_{\text {ren }}$ will be taken in the state $\left|\Psi_{i}\right\rangle$ defined by Eq. (6). Both the classical stress tensor and its quantum expectation value can be obtained by functionally differentiating, respectively, the classical action $S_{m}$ and the influence action $S_{\mathrm{IF}}$ with respect to the metric, according to Eqs. (A7) and (A8). The influence action describes the effect of the quantum matter fields on the gravitational field and results from functionally integrating the quantum matter fields. Note, however, that since our metric has been assumed to have the form $g_{\mu \nu}=$ $a^{2}(\eta) \eta_{\mu \nu}$, with the scale factor as the only independent kinematical degree of freedom, we can only functionally differentiate with respect to $a(\eta)$ and will just be able to obtain trace of the stress tensor, $T_{\mu}^{\mu}$. This can be seen as follows. Since $\delta g_{\mu \nu}=2 a \eta_{\mu \nu} \delta a=2 a^{-1} g_{\mu \nu} \delta a$, from Eq. (A7) we can write

$$
\begin{aligned}
\delta S_{m} & \equiv \int d^{n} x \frac{\delta S_{m}}{\delta g_{\mu \nu}} \delta g_{\mu \nu}=\int d^{n} x \sqrt{-g} T^{\mu \nu} g_{\mu \nu} \frac{\delta a}{a} \\
& =\int d \eta \int d^{n-1} x a^{n-1} T_{\mu}^{\mu} \delta a,
\end{aligned}
$$

so that $\delta S_{m} / \delta a=\mathcal{V} a^{n-1} T_{\mu}^{\mu}$, where the spatial comoving volume $\mathcal{V}$ appears as a consequence of the spatial homogeneity of the stress tensor. This also means that by functional derivation with respect to the scale factor we obtain, from the renormalized influence action $S_{\mathrm{IF}}^{\text {ren }}$ in Eq. (A8), the renormalized expectation value of the trace of the stresstensor operator:

$$
\left\langle\hat{T}_{\mu}^{\mu}\right\rangle=\left.\frac{1}{V a^{n-1}} \frac{\delta S_{\mathrm{IF}}^{\mathrm{ren}}\left[a^{+}, a^{-}\right]}{\delta a^{+}}\right|_{a^{+}=a^{-}=a},
$$

where from now on we will drop the subscript "ren" in the expectation value to simplify the notation.

In principle one could use either the 00 component of the semiclassical Einstein equation (the Friedmann equation) or the equation for the trace. There is, however, a subtle 
difference. For the sake of argument let us consider the classical limit only; whereas the classical Friedmann equation is a first-order differential equation in time for the scale factor the equation for the trace is of second order in time. The solutions of the Friedmann equation automatically satisfy the trace equation, but the Friedmann equation also constrains the initial conditions for the scale factor and its time derivative. If one works only with the trace equation, this additional information is missed. (When quantum corrections are added, higher-order derivatives appear in both equations, but we will explain how to deal with them in Sec. V.)

In this paper we will work with the semiclassical Friedmann equation. Therefore, we need to calculate $\left\langle\hat{T}_{00}\right\rangle$. One possibility is to start with a metric of the form $d s^{2}=-N^{2}(\tilde{\eta}) d \tilde{\eta}^{2}+a^{2}(\tilde{\eta}) \delta_{i j} d x^{i} d x^{j}$ with $N(\tilde{\eta})$ and $a(\tilde{\eta})$ independent, ${ }^{2}$ functionally differentiate with respect to $N$ and $a$, and finally take $N=a$ only after that. The functional derivative with respect to $N$ gives $\left\langle\hat{T}_{00}\right\rangle$ and the Friedmann equation. Alternatively, one can make use of a useful relation between $\left\langle\hat{T}_{00}\right\rangle$ and $\left\langle\hat{T}_{\mu}^{\mu}\right\rangle$ in a RW spacetime which is a consequence of the stress-tensor conservation law $\nabla_{\mu}\left\langle\hat{T}^{\mu \nu}\right\rangle=0$, and the fact that $\vec{\xi}=\partial / \partial \eta$ is a conformal Killing field, i.e., $2 \nabla_{(a} \xi_{b)}=\lambda g_{\mu \nu}$ with $\lambda=$ $2 \dot{a} / a$ in our case $[31,42]$. These two equations lead to

$$
\nabla_{\mu}\left(\left\langle\hat{T}^{\mu \nu}\right\rangle \xi_{\nu}\right)=\frac{\dot{a}}{a}\left\langle\hat{T}_{\mu}^{\mu}\right\rangle,
$$

which can be integrated over the spacetime volume bounded by the spacelike hypersurfaces corresponding to $\eta^{\prime}=-\infty$ and $\eta^{\prime}=\eta$ (for the construction introduced in Sec. II to generate the initial quantum state of the field the stress tensor is also conserved from $\eta^{\prime}=-\infty$ till our initial time $\eta^{\prime}=\eta_{i}$ ). Using Gauss's theorem we get a relationship between the integration of $\left\langle\hat{T}_{00}\right\rangle$ on the two hypersurfaces and the spacetime integral of $\left\langle\hat{T}_{\mu}^{\mu}\right\rangle$ which reads

$$
\left\langle\hat{T}_{00}(\eta)\right\rangle a^{n-2}(\eta)=C-\int_{-\infty}^{\eta} d \eta^{\prime} a^{n-1}\left(\eta^{\prime}\right) \dot{a}\left(\eta^{\prime}\right)\left\langle\hat{T}_{\mu}^{\mu}\left(\eta^{\prime}\right)\right\rangle,
$$

where $C=\left\langle\hat{T}_{00}(-\infty)\right\rangle a^{n-2}(-\infty)$ and we have divided by the spatial volume $\mathcal{V}$, which appears due to the spatial homogeneity of the stress-tensor expectation value. Since the constant $C$ is proportional to the expectation value of the energy density of a Minkowski vacuum, as follows from Eq. (6), it should vanish. Hence, from now on, we will take $C=0$.

\footnotetext{
${ }^{2}$ Provided that $N(\tilde{\eta})$ is nonvanishing and differentiable, such a metric can always be rewritten as $d s^{2}=a^{2}(\eta)\left(-d \eta^{2}+\right.$ $\delta_{i j} d x^{i} d x^{j}$ ) through a coordinate transformation involving a redefinition of the time $\tilde{\eta}$.
}

\section{B. The effective action}

In this section we compute the influence action needed to derive the expectation value of the trace of the stress tensor according to Eq. (10). Using the so-called CTP formalism, the influence action $S_{\mathrm{IF}}\left[g^{+}, g^{-}\right]$for an arbitrary metric is defined in Appendix A by Eqs. (A4) or (A10) for a general initial state of the field. Specializing Eq. (A10) to the conformally flat metric $g_{\mu \nu}=a^{2} \eta_{\mu \nu}$ with the definition of our initial state as given in Eq. (6), we get

$$
\begin{aligned}
e^{i S_{\mathrm{IF}}\left[a^{+}, a^{-}\right]}= & \int \mathcal{D} \varphi\left\langle 0,-\infty\left|\hat{U}_{-}\left(-\infty, \eta_{f}\right)\right| \varphi\right\rangle \\
& \times\left\langle\varphi\left|\hat{U}_{+}\left(\eta_{f},-\infty\right)\right| 0,-\infty\right\rangle,
\end{aligned}
$$

where $|\varphi\rangle$ are the properly normalized field eigenstates, such that $\hat{\varphi}(\vec{x})\left|\varphi\left(\vec{x}^{\prime}\right)\right\rangle=\varphi(\vec{x})\left|\varphi\left(\vec{x}^{\prime}\right)\right\rangle$, and the time evolution operator is

$$
\hat{U}_{ \pm}\left(\eta_{f},-\infty\right)=T \exp \left(-i \int_{-\infty}^{\eta_{f}} d \eta \hat{H}_{m}\left[a^{ \pm}, \hat{\pi}, \hat{\phi}\right]\right) .
$$

Using the path integral representation for the time evolution operator in terms of the action (4) for the scalar field we have

$$
e^{i S_{\mathrm{IF}}\left[a^{+}, a^{-}\right]}=\int \mathcal{D} \phi^{+} \mathcal{D} \phi^{-} e^{i\left(S_{m}\left[a^{+}, \phi^{+}\right]-S_{m}^{*}\left[a^{-}, \phi^{-}\right]\right)},
$$

where $\phi^{+}\left(\eta_{f}\right)=\phi^{-}\left(\eta_{f}\right)$. This expression corresponds to Eq. (A4) for an initial Minkowski vacuum state. To enforce this state we must take the usual $-i \epsilon$ prescription. Integrating by parts and taking into account that the action $S_{m}$ is quadratic in the field, we can write

$$
\begin{aligned}
e^{i S_{\mathrm{IF}}\left[a^{+}, a^{-}\right]} & =\int \mathcal{D} \phi^{+} \mathcal{D} \phi^{-} e^{(i / 2) \int d^{n} x\left(\phi^{+} A_{++} \phi^{+}+\phi^{-} A_{--} \phi^{-}\right)} \\
& =(\operatorname{det} A)^{-1 / 2}
\end{aligned}
$$

where the matrix $A$ is defined by $A_{++}=\eta^{\mu \nu} \partial_{\mu} \partial_{\nu}-$ $\nu\left(a^{+}\right)^{2} R^{+}+i \epsilon, A_{--}=-\left(\eta^{\mu \nu} \partial_{\mu} \partial_{\nu}-\nu\left(a^{-}\right)^{2} R^{-}-i \epsilon\right)$, and $A_{+-}=A_{-+}=0$, and a Gaussian integration has been performed in the last equality. Introducing the inverse matrix $G=A^{-1}$ we thus have

$$
S_{\mathrm{IF}}\left[a^{+}, a^{-}\right]=-\frac{i}{2} \operatorname{tr} \ln G .
$$

The matrix $G$ can be computed perturbatively. Following Refs. [43-45] we define $A=A^{0}+V$, where the matrix $V$ includes the time-dependent interaction with $V_{++}=-\nu\left(a^{+}\right)^{2} R^{+}, V_{--}=\nu\left(a^{-}\right)^{2} R^{-}$. Then up to second order in $\nu, G=G^{0}\left(1-V G^{0}+V G^{0} V G^{0}+\cdots\right)$ where $G^{0}$ is the Minkowski $2 \times 2$ CTP propagator with $G_{++}^{0}=\Delta_{F}, G_{--}^{0}=-\Delta_{D}, G_{+-}^{0}=-\Delta^{+}$, and $G_{-+}^{0}=$ $\Delta^{-}$, and where $\Delta_{F}$ and $\Delta_{D}$ are, respectively, the Feynman and the Dyson propagators and $\Delta^{ \pm}$are the Wightman functions: 


$$
\begin{aligned}
\Delta_{F / D}(x) & =-\int \frac{d^{n} p}{(2 \pi)^{n}} \frac{e^{i p \cdot x}}{p^{2} \mp i \epsilon}, \\
\Delta^{ \pm}(x) & = \pm 2 \pi i \int \frac{d^{n} p}{(2 \pi)^{n}} e^{i p \cdot x} \delta\left(p^{2}\right) \theta\left(\mp p^{0}\right) .
\end{aligned}
$$

Substituting into Eq. (17) we have (up to second order in $\nu$ )

$$
\begin{aligned}
S_{\mathrm{IF}}\left[a^{+}, a^{-}\right]= & -\frac{i}{2} \operatorname{tr} \ln G^{0}+\frac{i}{2} \operatorname{tr}\left(V_{++} \Delta_{F}\right) \\
& -\frac{i}{2} \operatorname{tr}\left(V_{--} \Delta_{D}\right)-\frac{i}{4} \operatorname{tr}\left(V_{++} \Delta_{F} V_{++} \Delta_{F}\right) \\
& -\frac{i}{4} \operatorname{tr}\left(V_{--} \Delta_{D} V_{--} \Delta_{D}\right) \\
& +\frac{i}{2} \operatorname{tr}\left(V_{++} \Delta^{+} V_{--} \Delta^{-}\right)
\end{aligned}
$$

The first three terms do not contribute to the dynamical equations for $a(\eta)$ : the first term is independent of $a$, and the second and third terms are tadpoles which are identically zero in dimensional regularization [46], so that there is no linear term in $\nu$ in the effective action. The fourth and fifth terms involve the product of Feynman and Dyson propagators and need regularization, whereas the last term is finite. Following closely Refs. $[43,44]$ we get, after expanding in powers of $(n-4)$, that the real part of $S_{\mathrm{IF}}$ in $n$ dimensions is

$$
\begin{aligned}
\operatorname{Re} S_{\mathrm{IF}}\left[a^{+}, a^{-}\right]= & -\frac{9 \nu^{2} V}{8 \pi^{2}}\left\{\frac{1}{n-4} \int d \eta \Delta\left(\left(\frac{\ddot{a}}{a}\right)^{2}\right)\right. \\
& +\frac{1}{3} \int d \eta \Delta\left(\frac{\ddot{a}}{a}\left[3\left(\frac{\dot{a}}{a}\right)^{2}+2 \frac{\ddot{a}}{a}\right]\right) \\
& +2 \iint d \eta d \eta^{\prime} \Delta\left(\frac{\ddot{a}}{a}(\eta)\right) \tilde{H}\left(\eta-\eta^{\prime}\right) \\
& \left.\times \Sigma\left(\frac{\ddot{a}}{a}\left(\eta^{\prime}\right)\right)\right\}+O(n-4),
\end{aligned}
$$

where we have used the difference and semisum notations $\Delta(f) \equiv f^{+}-f^{-}$and $\Sigma(f) \equiv\left(f^{+}+f^{-}\right) / 2$, respectively, and the kernel $\tilde{H}\left(\eta-\eta^{\prime}\right)$ is given by

$$
\begin{aligned}
\tilde{H}\left(\eta-\eta^{\prime}\right)= & \int \frac{d \omega}{2 \pi} e^{-i \omega\left(\eta-\eta^{\prime}\right)}\left(\ln |\omega|+\frac{i \pi}{2} \operatorname{sgn}(-\omega)\right. \\
& \left.-\frac{1}{2}(2+\ln 4 \pi-\gamma)\right)
\end{aligned}
$$

where $\gamma$ is the Euler-Mascheroni constant. Note that although the argument of the logarithm is not dimensionless, when combining the influence action with the counterterms in the bare gravitational action, the contribution involving the renormalization scale $\mu$ will finally render the argument of the logarithm dimensionless, as we will see below. There is also an imaginary part in $S_{\mathrm{IF}}$, but it does not contribute to the expectation value (10), and thus to the semiclassical equation for $a$, because it depends quadratically on the difference variable $\Delta(\ddot{a} / a)$. This means that when functionally deriving with respect to $a^{+}$and then taking $a^{+}=a^{-}=a$ to get the expectation value, the imaginary contribution vanishes, as it should. The role of the imaginary part of $S_{\mathrm{IF}}$ is related to the so-called noise kernel, which accounts for the fluctuations of the stress tensor and allows to go beyond the semiclassical equations, which capture only the averaged value of the stress tensor. The noise kernel plays a key role in stochastic gravity; see Refs. [47-51] for the general theory and Refs. [45,52-55] for cosmological applications.

As explained in Appendix A, the dynamical equations for the gravitational field can be derived from the so-called CTP effective action, $\Gamma\left[a^{+}, a^{-}\right]$, the two ingredients of which are the gravitational action $S_{g}[a]$, given by Eq. (8), and the influence action $S_{\mathrm{IF}}\left[a^{+}, a^{-}\right]$. Specializing Eq. (A5) to the conformally flat metric in Eq. (1), the regularized CTP effective action becomes

$$
\Gamma\left[a^{+}, a^{-}\right]=S_{g}\left[a^{+}\right]-S_{g}\left[a^{-}\right]+S_{\mathrm{IF}}\left[a^{+}, a^{-}\right] .
$$

The real part of the regularized influence action is given by Eq. (20) and diverges for $n=4$. Thus, we need to add appropriate local covariant counterterms, which we denoted by $S_{g}^{c}[a]$, to the bare gravitational action. For a massless field only counterterms quadratic in the curvature, as explicitly given by Eq. (A12), are needed. The integrand of the first term on the right-hand side of Eq. (A12), which is independent of $\nu^{2}$, is proportional to the square of the Weyl tensor in $n=4$ dimensions. For a conformally flat metric like the metric (1) that we are considering here, the Weyl tensor vanishes and thus this term vanishes in four dimensions. However, it plays a crucial role in the trace anomaly [31]. In fact, expanding in powers of $(n-4)$ and neglecting a total divergence, we have

$$
\begin{aligned}
& \mu^{n-4} \int d^{n} x \sqrt{-g}\left(R_{\mu \nu \rho \sigma} R^{\mu \nu \rho \sigma}-R_{\mu \nu} R^{\mu \nu}\right) \\
& =-V(n-4) \int d \eta\left[3\left(\frac{\ddot{a}}{a}\right)^{2}-\left(\frac{\dot{a}}{a}\right)^{4}\right]+O\left((n-4)^{2}\right),
\end{aligned}
$$

which is of order $O(n-4)$ and therefore gives a finite contribution when multiplied by the divergent $(n-4)^{-1}$ factor. On the other hand, due to the $O(n-4)$ dependence there will be no contribution proportional to the parameter $\alpha$ from Eq. (A12), as expected since the tensor $A_{\mu \nu}$ in Eq. (A1) vanishes for a conformally flat metric. As for the second term on the right-hand side of Eq. (A12), we have

$$
\begin{aligned}
\mu^{n-4} \int d^{n} x \sqrt{-g} R^{2}= & V \int d \eta\left\{36\left(\frac{\ddot{a}}{a}\right)^{2}+12(n-4)\right. \\
& \times\left\{3\left(\frac{\ddot{a}}{a}\right)^{2} \ln (a \mu)\right. \\
& \left.\left.+\frac{\ddot{a}}{a}\left[3\left(\frac{\dot{a}}{a}\right)^{2}+2 \frac{\ddot{a}}{a}\right]\right\}\right\} \\
& +O\left((n-4)^{2}\right)
\end{aligned}
$$


When multiplied by $(n-4)^{-1}$, the first term of this expansion cancels out the $(n-4)^{-1}$ divergence of Eq. (20).

Finally, we can add the regularized counterterms of Eq. (A12) with the particular values in Eqs. (23) and (24) to the Einstein-Hilbert action (including the cosmological constant) to obtain the total bare gravitational action $S_{g}[a]$. Together with the regularized influence action, whose real part is given by Eq. (20), it gives the regularized CTP effective action $\Gamma\left[a^{+}, a^{-}\right]$. We can then take the limit $n \rightarrow$ 4 to obtain the four-dimensional effective action in terms of the renormalized gravitational action and influence action:

$$
\Gamma\left[a^{+}, a^{-}\right]=S_{g}^{\mathrm{ren}}\left[a^{+}\right]-S_{g}^{\mathrm{ren}}\left[a^{-}\right]+S_{\mathrm{IF}}^{\mathrm{ren}}\left[a^{+}, a^{-}\right] .
$$

The result for the renormalized real part of the influence action in four dimensions is

$$
\begin{aligned}
\operatorname{Re} S_{\mathrm{IF}}^{\mathrm{ren}}\left[a^{+}, a^{-}\right]= & \mathcal{V} \int d \eta\left\{-\frac{1}{2880 \pi^{2}} \Delta\left(3\left(\frac{\ddot{a}}{a}\right)^{2}-\left(\frac{\dot{a}}{a}\right)^{4}\right)\right. \\
& \left.+\frac{9 \nu^{2}}{8 \pi^{2}}\left[\Delta\left(\left(\frac{\ddot{a}}{a}\right)^{2} \ln a\right)-2 \int d \eta^{\prime} \Delta\left(\frac{\ddot{a}}{a}(\eta)\right) H\left(\eta-\eta^{\prime} ; \bar{\mu}\right) \Sigma\left(\frac{\ddot{a}}{a}\left(\eta^{\prime}\right)\right)\right]\right\}
\end{aligned}
$$

where we have incorporated the renormalization scale $\bar{\mu}=$ $\mu \exp [(2+\ln 4 \pi-\gamma) / 2]$ in the new kernel

$$
H\left(\eta-\eta^{\prime} ; \bar{\mu}\right)=\int \frac{d \omega}{2 \pi} e^{-i \omega\left(\eta-\eta^{\prime}\right)}\left(\ln \frac{|\omega|}{\bar{\mu}}+\frac{i \pi}{2} \operatorname{sgn}(-\omega)\right) .
$$

From the action in Eq. (26) one can obtain the renormalized expectation value of the trace of the stress tensor (remember that the imaginary part of the influence action plays no role in that). Similarly, by taking the functional derivative of the CTP effective action $\Gamma\left[a^{+}, a^{-}\right]$with respect to $a^{+}$and then equating $a^{+}=a^{-}=a$ we obtain the trace of the semiclassical Einstein equation. Note that the CTP effective action, given by Eq. (22) or equivalently by Eq. (25), is renormalization-group invariant, i.e., it is independent of the renormalization-group scale $\mu$, and so are the physical predictions that one can derive from it. The dependence on $\mu$ in $\operatorname{Re} S_{\mathrm{IF}}^{\mathrm{ren}}\left[a^{+}, a^{-}\right]$, which gives rise to a local term of the form $\left(9 \nu^{2} / 8 \pi^{2}\right) \ln \mu \Delta\left((\ddot{a} / a)^{2}\right)$, is exactly compensated by the dependence on $\mu$ of the renormalized parameter $\beta$ multiplying the $R^{2}$ term in the renormalized gravitational action. This can be traced back to the fact that the bare parameter $\beta_{B}$ is independent of $\mu$, as explained in Appendix A.

We close this section by mentioning an alternative (but entirely equivalent) method of calculating the influence action provided in Ref. [56]. The approach, which is based on decomposing the field in spatial Fourier modes, computing the unitary evolution operator for each mode perturbatively in the interaction picture, and summing over all the modes at the end, can be useful when considering more general initial states at a finite initial time $\eta_{i}$ which are not necessarily of the form given by Eq. (6).

\section{THE EXPECTATION VALUE OF THE STRESS TENSOR}

\section{A. The trace}

Functionally differentiating with respect to $a^{+}$the expression for the influence action given by Eq. (26) and using Eq. (10), we obtain the expectation value of the trace of the stress tensor:

$$
\begin{aligned}
\left\langle\hat{T}_{\mu}^{\mu}\right\rangle= & \frac{1}{a^{3}}\left\{-\frac{6}{2880 \pi^{2}}\left[\frac{d^{2}}{d \eta^{2}}\left(\frac{\ddot{a}}{a^{2}}\right)-\frac{\ddot{a}^{2}}{a^{3}}\right]\right. \\
& -\frac{4}{2880 \pi^{2}}\left[\frac{d}{d \eta}\left(\frac{\dot{a}^{3}}{a^{4}}\right)+\frac{\dot{a}^{4}}{a^{5}}\right] \\
& +\frac{9 \nu^{2}}{4 \pi^{2}}\left[\frac{d^{2}}{d \eta^{2}}\left(\frac{\ddot{a}}{a^{2}} \ln a\right)-\frac{\ddot{a}^{2}}{a^{3}}\left(\ln a-\frac{1}{2}\right)\right. \\
& \left.\left.-\frac{d^{2}}{d \eta^{2}}\left(\frac{1}{a} \kappa\left[\frac{\ddot{a}}{a} ; \eta\right)\right)+\frac{\ddot{a}}{a^{2}} \kappa\left[\frac{\ddot{a}}{a} ; \eta\right)\right]\right\},
\end{aligned}
$$

where $\kappa[f ; \eta)=\int_{-\infty}^{\infty} d \eta^{\prime} H\left(\eta-\eta^{\prime} ; \bar{\mu}\right) f\left(\eta^{\prime}\right)$. One can easily check that the usual result for the trace anomaly is obtained in the conformal limit $\nu \rightarrow 0$. Indeed, taking into account that in four dimensions

$$
\square R=-\frac{6}{a^{3}}\left[\frac{d^{2}}{d \eta^{2}}\left(\frac{\ddot{a}}{a^{2}}\right)-\frac{\ddot{a}^{2}}{a^{3}}\right]
$$

and

$$
R_{\mu \nu \rho \sigma} R^{\mu \nu \rho \sigma}-R_{\mu \nu} R^{\mu \nu}=-\frac{4}{a^{3}}\left[\frac{d}{d \eta}\left(\frac{\dot{a}^{3}}{a^{4}}\right)+\frac{\dot{a}^{4}}{a^{5}}\right],
$$

Eq. (28) can be rewritten as

$$
\begin{aligned}
\left\langle\hat{T}_{\mu}^{\mu}\right\rangle= & \frac{1}{2880 \pi^{2}} \square R+\frac{1}{2880 \pi^{2}}\left(R_{\mu \nu \rho \sigma} R^{\mu \nu \rho \sigma}-R_{\mu \nu} R^{\mu \nu}\right) \\
& +O\left(\nu^{2}\right),
\end{aligned}
$$

which coincides with the trace anomaly [31] for a massless conformal scalar field when $\nu=0$. Note that the counterterm in Eq. (23), when multiplied by the divergent factor $(n-4)^{-1}$, plays a key role for this result.

Equation (28) has a nonlocal term which includes the functional $\kappa[\ddot{a} / a ; \eta)$ and its first and second derivatives. Let us examine this nonlocal part in some detail. The Fourier transform in Eq. (27) can be computed [see Eq. (VII.7.18) in Ref. [57]] to yield 
$H\left(\eta-\eta^{\prime} ; \bar{\mu}\right)=-\mathcal{P} f \frac{\theta\left(\eta-\eta^{\prime}\right)}{\eta-\eta^{\prime}}-(\gamma+\ln \bar{\mu}) \delta\left(\eta-\eta^{\prime}\right)$,

where $\mathcal{P} f$ stands for Hadamard's finite part prescription, and $\gamma$ is the Euler-Mascheroni constant. This prescription means that

$$
\begin{aligned}
\kappa[f ; \eta)= & -\lim _{\epsilon \rightarrow 0^{+}}\left\{\int_{-\infty}^{\eta-\epsilon} \frac{d \eta^{\prime}}{\eta-\eta^{\prime}} f\left(\eta^{\prime}\right)\right. \\
& +(\ln \epsilon+\ln \bar{\mu}+\gamma) f(\eta)\} .
\end{aligned}
$$

Since the evolution from $\eta=-\infty$ to $\eta_{i}$ can be regarded as an auxiliary way to generate the initial state of the quantum field, which is determined by the scale factor $a(\eta)$ for times $\eta \leq \eta_{i}$ and denoted earlier by $a_{\Psi}(\eta)$, it is convenient to define $v(\eta) \equiv\left(\ddot{a}_{\Psi} / a_{\Psi}\right)(\eta)$. Therefore, for $\eta \leq \eta_{i}$ we have $\kappa\left[\ddot{a}_{\Psi} / a_{\Psi} ; \eta\right)=\kappa[v ; \eta)$, whereas for $\eta>\eta_{i}$ the integral in Eq. (33) can be separated into two parts:

$$
\kappa\left[\frac{\ddot{a}}{a} ; \eta\right)=\kappa_{1}\left[\frac{\ddot{a}}{a} ; \eta\right)+\kappa_{2}[v ; \eta),
$$

where

$$
\begin{aligned}
\kappa_{1}\left[\frac{\ddot{a}}{a} ; \eta\right)= & -\lim _{\epsilon \rightarrow 0^{+}}\left\{\int_{\eta_{i}}^{\eta-\epsilon} \frac{d \eta^{\prime}}{\eta-\eta^{\prime}} \frac{\ddot{a}}{a}\left(\eta^{\prime}\right)\right. \\
& \left.+(\ln \epsilon+\ln \bar{\mu}+\gamma) \frac{\ddot{a}}{a}(\eta)\right\},
\end{aligned}
$$

which involves a time integration only after $\eta_{i}$, and

$$
\kappa_{2}[v ; \eta)=-\int_{-\infty}^{\eta_{i}} \frac{d \eta^{\prime}}{\eta-\eta^{\prime}} v\left(\eta^{\prime}\right),
$$

which involves a time integration only before $\eta_{i}$. Following this notation one can also separate the trace in Eq. (28) for $\eta>\eta_{i}$ into two parts: $\left\langle\hat{T}_{\mu}^{\mu}\right\rangle=\left\langle\hat{T}_{\mu}^{\mu}\right\rangle_{1}+\left\langle\hat{T}_{\mu}^{\mu}\right\rangle_{2}$, where the first term involves $\kappa_{1}[\ddot{a} / a ; \eta)$ and the second one involves $\kappa_{2}[v ; \eta)$. From Eqs. (5) and (6), and taking into account that in four dimensions $a^{2} R=6 \ddot{a} / a$, we see that $v(\eta)$ completely determines the initial state of the quantum field. Thus, $\left\langle\hat{T}_{\mu}^{\mu}\right\rangle_{1}$ is state independent, whereas $\left\langle\hat{T}_{\mu}^{\mu}\right\rangle_{2}$ contains all the dependence on the initial state. In fact, the former will appear in the trace even if the initial state does not have the form given by Eq. (6).

\section{B. The initial conditions}

We can now see that the state-independent part of the trace of the stress tensor, namely $\left\langle\hat{T}_{\mu}^{\mu}\right\rangle_{1}$, diverges in the limit $\eta \rightarrow \eta_{i}^{+}$, corresponding to the initial time. Let us consider the definition of $\kappa_{1}[\ddot{a} / a ; \eta)$ in Eq. (35) and Taylor expand $\ddot{a} / a$ around $\eta^{\prime}=\eta$ so that $(\ddot{a} / a)\left(\eta^{\prime}\right)=$ $(\ddot{a} / a)(\eta)+O\left(\eta-\eta^{\prime}\right)$; we then have

$$
\begin{aligned}
\int_{\eta_{i}}^{\eta-\epsilon} \frac{d \eta^{\prime}}{\eta-\eta^{\prime}} \frac{\ddot{a}}{a}\left(\eta^{\prime}\right)= & -\frac{\ddot{a}}{a}(\eta) \ln \epsilon+\frac{\ddot{a}}{a}(\eta) \ln \left(\eta-\eta_{i}\right) \\
& +O\left(\eta-\eta_{i}\right),
\end{aligned}
$$

which implies that

$$
\begin{aligned}
\kappa_{1}\left[\frac{\ddot{a}}{a} ; \eta\right)= & -\frac{\ddot{a}}{a}(\eta)\left[\ln \left(\eta-\eta_{i}\right)+\ln \bar{\mu}+\gamma\right] \\
& +O\left(\eta-\eta_{i}\right),
\end{aligned}
$$

so that $\kappa_{1}[\ddot{a} / a ; \eta)$ is finite for all $\eta>\eta_{i}$, but diverges when $\eta \rightarrow \eta_{i}^{+}$. Moreover, in addition to $\kappa_{1}[\ddot{a} / a ; \eta),\left\langle\hat{T}_{\mu}^{\mu}\right\rangle_{1}$ also contains the first and second time derivatives of $\kappa_{1}$. Obviously upon derivation Eq. (38) becomes more singular at $\eta \rightarrow \eta_{i}^{+}$, and in general the divergences of the three singular terms will not cancel out. Therefore, $\left\langle\hat{T}_{\mu}^{\mu}\right\rangle_{1} \rightarrow \infty$ in the limit $\eta \rightarrow \eta_{i}^{+}$.

Thus, $\left\langle\hat{T}_{\mu}^{\mu}\right\rangle$ will diverge at the initial time unless we choose an initial state that cancels the previous divergences. This partly motivates the class of initial states we consider in this paper. Taylor expanding $v$ around $\eta^{\prime}=$ $\eta_{i}$, and following a procedure analogous to the one that led to Eq. (37) [notice that condition (7) ensures that $\kappa_{2}[v ; \eta$ ) is well behaved in its lower limit], one can check that

$$
\kappa_{2}[v ; \eta)=v\left(\eta_{i}\right) \ln \left(\eta-\eta_{i}\right)+\text { finite terms, }
$$

where by "finite terms" we mean terms which are finite in the limit $\eta \rightarrow \eta_{i}$. Therefore, $\kappa[\ddot{a} / a ; \eta)=\kappa_{1}[\ddot{a} / a ; \eta)+$ $\kappa_{2}[v ; \eta)$ is finite at $\eta_{i}$ provided that $v\left(\eta_{i}\right)=$ $\lim _{\eta \rightarrow \eta_{i}^{+}}(\ddot{a} / a)$. If this condition is satisfied, it is easy to see from Eqs. (35) and (36) that $(d / d \eta) \kappa[\ddot{a} / a ; \eta)=$ $\kappa[d(\ddot{a} / a) / d \eta ; \eta)$. Thus, by iterating the same argument we find that the conditions that an initial state of the class (6) must satisfy in order to avoid initial time divergences of $\left\langle\hat{T}_{\mu}^{\mu}\right\rangle$ are

$$
\begin{gathered}
v\left(\eta_{i}\right)=\lim _{\eta \rightarrow \eta_{i}^{+}}\left(\frac{\ddot{a}}{a}\right), \quad \dot{v}\left(\eta_{i}\right)=\lim _{\eta \rightarrow \eta_{i}^{+}} \frac{d}{d \eta}\left(\frac{\ddot{a}}{a}\right), \\
\ddot{v}\left(\eta_{i}\right)=\lim _{\eta \rightarrow \eta_{i}^{+}} \frac{d^{2}}{d \eta^{2}}\left(\frac{\ddot{a}}{a}\right) .
\end{gathered}
$$

In other words, the cosmological scale factors before and after the initial time must be matched with continuity up to the fourth derivative. Equations (40) have been presented as conditions on the preparation of the initial state given a scale factor $a(\eta)$ for $\eta \geq \eta_{i}$. Alternatively, one can regard these equations as initial conditions on the scale factor given some initial state defined by Eq. (6). Henceforth we will assume that these conditions are satisfied.

\section{The energy density}

Once the expectation value of the trace of the stress tensor is known, the 00 component of the stress tensor may be obtained from Eq. (12). The local part of $\dot{a} a^{3}\left\langle\hat{T}_{\mu}^{\mu}\right\rangle$ 
is a derivative, so it gives rise to local terms in $a^{2}\left\langle\hat{T}_{00}\right\rangle$. The result, for $\eta>\eta_{i}$, is

$$
\begin{aligned}
a^{2}\left\langle\hat{T}_{00}\right\rangle= & \frac{6}{2880 \pi^{2}}\left[\dot{a} \frac{d}{d \eta}\left(\frac{\ddot{a}}{a^{2}}\right)-\frac{1}{2}\left(\frac{\ddot{a}}{a}\right)^{2}\right]+\frac{3}{2880 \pi^{2}}\left(\frac{\dot{a}}{a}\right)^{4} \\
& -\frac{9 \nu^{2}}{4 \pi^{2}}\left\{\ln a\left[\dot{a} \frac{d}{d \eta}\left(\frac{\ddot{a}}{a^{2}}\right)-\frac{1}{2}\left(\frac{\ddot{a}}{a}\right)^{2}\right]+\frac{\dot{a}^{2} \ddot{a}}{a^{3}}\right. \\
& +T[a, v]\},
\end{aligned}
$$

where $T$, which includes the nonlocal part of this expectation value and comes from integrating the last two terms in Eq. (28), is defined by

$$
\begin{aligned}
T[a, v ; \eta)= & \int_{-\infty}^{\eta} d \eta^{\prime} \dot{a}\left[-\frac{d^{2}}{d \eta^{\prime 2}}\left(\frac{1}{a} \kappa\left[\frac{\ddot{a}}{a} ; \eta^{\prime}\right)\right)\right. \\
& \left.+\frac{\ddot{a}}{a^{2}} \kappa\left[\frac{\ddot{a}}{a} ; \eta^{\prime}\right)\right] .
\end{aligned}
$$

Making use of the "smoothness" of the scale factor at $\eta_{i}$ assumed above, and following the previous notation for the separation of the nonlocal terms, we can again write

$$
T[a, v]=T_{1}[a]+T_{2}[a, v],
$$

where we split $T[a, v]$ into two parts: one independent of $v$ and a second one which contains the entire dependence on $v$. This is achieved by splitting the integral in Eq. (42) as well as the functional $\kappa$. The first part is given by

$$
\begin{aligned}
T_{1}[a ; \eta)= & \int_{\eta_{i}}^{\eta} d \eta^{\prime}\left(\frac{\dot{a}}{a}\right)\left(\eta^{\prime}\right)\left\{2 \frac{d}{d \eta^{\prime}}\left(\frac{\dot{a}}{a}\right) \kappa_{1}\left[\frac{\ddot{a}}{a} ; \eta^{\prime}\right)\right. \\
& +2\left(\frac{\dot{a}}{a}\right)\left(\eta^{\prime}\right) \kappa_{1}\left[\frac{d}{d \eta^{\prime \prime}}\left(\frac{\ddot{a}}{a}\right) ; \eta^{\prime}\right) \\
& \left.-\kappa_{1}\left[\frac{d^{2}}{d \eta^{\prime 2}}\left(\frac{\ddot{a}}{a}\right) ; \eta^{\prime}\right)\right\} .
\end{aligned}
$$

This is obtained from Eq. (42) by explicitly applying the second-order derivative $d^{2} / d \eta^{\prime 2}$ and taking into account that when the conditions in Eqs. (40) hold, the derivatives acting on the functional $\kappa$ can be taken inside and applied to the argument. All this is done before splitting the integral and the functional $\kappa$. Similarly, the second part in Eq. (43) can be written as

$$
\begin{aligned}
T_{2}[a, v ; \eta)= & \int_{\eta_{i}}^{\eta} d \eta^{\prime}\left(\frac{\dot{a}}{a}\right)\left(\eta^{\prime}\right)\left\{2 \frac{d}{d \eta^{\prime}}\left(\frac{\dot{a}}{a}\right) \kappa_{2}\left[v ; \eta^{\prime}\right)\right. \\
& \left.+2\left(\frac{\dot{a}}{a}\right)\left(\eta^{\prime}\right) \kappa_{2}\left[\dot{v} ; \eta^{\prime}\right)-\kappa_{2}\left[\ddot{v} ; \eta^{\prime}\right)\right\} \\
& +\left(\frac{\dot{a}}{a}\right)^{2}\left(\eta_{i}\right) \kappa\left[v ; \eta_{i}\right)-\left(\frac{\dot{a}}{a}\right)\left(\eta_{i}\right) \kappa\left[\dot{v} ; \eta_{i}\right) \\
& +\int_{-\infty}^{\eta_{i}} d \eta^{\prime} v\left(\eta^{\prime}\right) \kappa\left[\dot{v} ; \eta^{\prime}\right) .
\end{aligned}
$$

The different form of the last three terms, which correspond to the integral from $-\infty$ to $\eta_{i}$, is because we made use of the following equivalent expression for the last two terms in Eq. (28) times $\left(-\dot{a} a^{3}\right)$ :

$$
\begin{aligned}
- & \dot{a} \frac{d^{2}}{d \eta^{\prime 2}}\left(\frac{1}{a} \kappa\left[\frac{\ddot{a}}{a} ; \eta^{\prime}\right)\right)+\dot{a} \frac{\ddot{a}}{a^{2}} \kappa\left[\frac{\ddot{a}}{a} ; \eta^{\prime}\right) \\
& =-\frac{d}{d \eta^{\prime}}\left(\dot{a} \frac{d}{d \eta^{\prime}}\left(\frac{1}{a} \kappa\left[\frac{\ddot{a}}{a} ; \eta^{\prime}\right)\right)\right)+\frac{\ddot{a}}{a} \frac{d}{d \eta^{\prime}} \kappa\left[\frac{\ddot{a}}{a} ; \eta^{\prime}\right) .
\end{aligned}
$$

In addition to being convenient later on, we did this so that it became manifest that $T_{2}[a, v]$ depends on $a_{\Psi}$ only through $v$. From Eqs. (44) and (45) it is clear that all the dependence of the energy density on the initial state of the quantum field is included in $T_{2}[a, v]$. We should, however, remember that conditions (40) have been used so that the divergences from the evolution after $\eta_{i}$ and those from the initial state would cancel out. Consequently, the integrand on the right-hand side of Eq. (44) and the integrand of the first integral in Eq. (45) only exhibit logarithmic divergences when $\eta^{\prime} \rightarrow \eta_{i}$ which would cancel out when adding $T_{1}$ and $T_{2}$. In fact, they give a finite contribution even separately since, being logarithmic, they are finite upon integration.

The energy density that we have just computed is valid for any scale factor at $\eta \geq \eta_{i}$ and an initial state corresponding to any regular function $v(\eta)$ which satisfies conditions (7) and (40). It can be included in the semiclassical Friedmann equation in the presence of any other classical source. In this paper we concentrate on the case where the classical source is a cosmological constant.

\section{THE SEMICLASSICAL FRIEDMANN EQUATION}

\section{A. The equation}

Next, we proceed to analyze the evolution of the scale factor after the initial time $\eta_{i}$, when it is driven by a cosmological constant and a free massless scalar field nonconformally coupled to the curvature. We will use the 00 component of the semiclassical Einstein equation (A1), namely, $G_{00}=-\Lambda g_{00}-\beta B_{00}+\kappa\left\langle\hat{T}_{00}\right\rangle_{\text {ren. }}$. Note that we have already taken into account that $A_{\mu \nu}=0$ in our conformally flat spacetime. On the other hand, evaluating $B_{\mu \nu}$, as given by Eq. (A3), for the RW metric (1), we get

$$
B_{00}=-\frac{72}{a^{2}}\left[\dot{a} \frac{d}{d \eta}\left(\frac{\ddot{a}}{a^{2}}\right)-\frac{1}{2}\left(\frac{\ddot{a}}{a}\right)^{2}\right],
$$

which can also be obtained by using Eq. (24) for $n=4$ and noticing that the term proportional to $\Delta(\ddot{a} / a)$ gives rise to the first term on the right-hand side of Eq. (41). Taking into account that $G_{00}=3(\dot{a} / a)^{2}$ for the metric (1) and using Eq. (41) we obtain the following expression for the semiclassical Friedmann equation: 


$$
\begin{aligned}
\dot{a}^{2}= & H^{2} a^{4}+\frac{l_{p}^{2}}{3}\left\{-\tilde{\beta}\left[\dot{a} \frac{d}{d \eta}\left(\frac{\ddot{a}}{a^{2}}\right)-\frac{1}{2}\left(\frac{\ddot{a}}{a}\right)^{2}\right]+\frac{3}{360 \pi}\left(\frac{\dot{a}}{a}\right)^{4}\right. \\
& \left.-\frac{18 \nu^{2}}{\pi}\left\{\ln a\left[\dot{a} \frac{d}{d \eta}\left(\frac{\ddot{a}}{a^{2}}\right)-\frac{1}{2}\left(\frac{\ddot{a}}{a}\right)^{2}\right]+\frac{\dot{a}^{2} \ddot{a}}{a^{3}}+T[a, v]\right\}\right\},
\end{aligned}
$$

where $\tilde{\beta}=576 \pi \beta-1 /(60 \pi)$, and we have introduced the Hubble constant $H \equiv(\Lambda / 3)^{1 / 2}$ and the Planck length $l_{p}=\sqrt{G}=(\kappa / 8 \pi)^{1 / 2}$. These two constants introduce two different time scales into the problem, the Hubble time $H^{-1}$ and the Planck time $t_{p}=l_{p}$, which we will assume to be well separated, namely, $H^{-1} \gg l_{p}$. It should also be emphasized that the semiclassical Friedmann equation (48) is invariant under conformal-time translations in the following sense. We already know that $v(\eta)$, with domain $\left(-\infty, \eta_{i}\right]$, characterizes the initial state of the quantum field, $\left|\Psi\left(\eta_{i}\right)\right\rangle$, when it is of the form given by Eq. (6). The time translation of $v(\eta)$, given by $v_{\Delta}(\eta)=v(\eta+\Delta)$, is defined in the domain $\left(-\infty, \eta_{i}-\Delta\right]$ and corresponds to an initial state $\left|\Psi_{\Delta}\left(\eta_{i}-\Delta\right)\right\rangle=\left|\Psi\left(\eta_{i}\right)\right\rangle$. It is then easy to see from Eq. (48) that if $a(\eta)$ is a solution for some initial state $\left|\Psi\left(\eta_{i}\right)\right\rangle$ characterized by $v(\eta)$, then $a(\eta+\Delta)$ is a solution for the initial state $\left|\Psi_{\Delta}\left(\eta_{i}-\Delta\right)\right\rangle$, characterized by $v_{\Delta}(\eta)$.

Our semiclassical Friedmann equation (48), which is a nonlinear third-order integro-differential equation, looks like a typical backreaction equation. Because of the higher-order time derivatives, those equations exhibit extra degrees of freedom which usually translate into unphysical runaway-type solutions [58]. [Indeed, if we had proceeded analogously to what was done in Ref. [59] for QED, the Friedmann equation and the equation for the trace would fix the third and fourth derivatives of the scale factor at the initial time, given some freely specified initial values for $a$, $\dot{a}$, and $\ddot{a}$. This is in contrast to the classical case, where only $a$ (or alternatively $\dot{a}$ ) can be specified independently at the initial time.] To get rid of the unphysical solutions several methods have been proposed. In some methods one looks only for analytic solutions, in a suitable perturbative parameter, in order to select physical solutions only [60-62]. In some other methods the equation itself is changed in order to get rid of the higher-order derivatives; this is the case of the so-called order-reduction method [63]. See Ref. [33] for a review of the advantages and the shortcomings of the different methods in the context of semiclassical gravity.

Given how we have proceeded in this paper, it is more natural in our case to interpret the point discussed in the previous paragraph as follows. The solutions of our semiclassical Friedmann equation (48) must satisfy the three initial conditions in Eqs. (40). This means that given some initial state, completely characterized by the function $v(\eta)$, the semiclassical Friedmann equation together with the equation for the trace fix $a\left(\eta_{i}\right)$ and $\dot{a}\left(\eta_{i}\right)$ since the second, third, and fourth derivatives at the initial time are fixed by Eqs. (40). Thus, there is in general a unique solution compatible with a given initial state. Certain initial states, however, give rise to solutions with characteristic time scales of the order of the Planck time (corresponding to exponential growth or oscillatory behavior), which lie beyond the regime of validity of the low-energy effective field theory approach that we have implicitly been using. In those circumstances the higher-order corrections involving terms with positive powers of the curvature and suppressed by the corresponding powers of the Planck mass can no longer be neglected and the low-energy expansion breaks down (since there are in principle an infinite number of such terms). We will consider only situations with no Planckian features where higher-order corrections are negligible. Hence, in the spirit of the effective field theory approach (valid for $l_{p} H \ll 1$ ), we will look for perturbative solutions ${ }^{3}$ in powers of $\left(l_{p} H\right)^{2}$ as

$$
a(\eta)=a_{0}(\eta)+\left(l_{p} H\right)^{2} a_{1}(\eta)+O\left(\left(l_{p} H\right)^{4}\right),
$$

where $a_{0}(\eta)$ satisfies the classical Friedmann equation. From now on we will use the subscript 0 to indicate the classical unperturbed values. Without loss of generality we can focus on the solutions $a(\eta)$ with $a_{0}(\eta)=-1 / H \eta$ defined for $\eta_{i}<\eta<0$ since any other well-behaved solution not involving Planckian scales is connected to one of these by a time translation. The function characterizing the initial state is given by $v(\eta)=v_{0}(\eta)+O\left(\left(l_{p} H\right)^{2}\right)$, and the conditions in Eqs. (40) imply

$$
v_{0}\left(\eta_{i}\right)=\frac{2}{\eta_{i}^{2}}, \quad \dot{v}_{0}\left(\eta_{i}\right)=-\frac{4}{\eta_{i}^{3}}, \quad \ddot{v}_{0}\left(\eta_{i}\right)=\frac{12}{\eta_{i}^{4}}
$$

Substituting the expression (49) into the semiclassical Friedmann equation (48), we obtain an equation for the first-order perturbation $a_{1}(\eta)$ :

\footnotetext{
${ }^{3}$ A perturbative expansion may sometimes miss the right longtime behavior of the semiclassical solution. This can happen when the effect of the quantum corrections, although locally small, builds up over long times giving rise to substantial deviations from the classical solution. An example of such a situation is the evolution of a black hole spacetime when the backreaction of the emitted Hawking radiation is taken into account. One possibility in those cases is to modify the backreaction equation using an order-reduction procedure and then solve the resulting equation nonperturbatively [64]. In the cosmological case considered here one can argue that such an accumulation effect will not be present. That is because the classical cosmological constant implies a monotonous growing behavior for the unperturbed solution and the locally small effect due to the vacuum polarization of the quantum fields (much smaller than the classical cosmological constant) generates a perturbation which is always small compared to the unperturbed solution. Moreover, one can explicitly check a posteriori that the deviation from the classical solution does not become significant at late times.
} 


$$
\dot{a}_{1}=-\frac{2}{\eta} a_{1}+\frac{1}{H}\left\{\frac{1}{720 \pi} \frac{1}{\eta^{2}}-\frac{3 \nu^{2}}{\pi}\left[\frac{2}{\eta^{2}}+\eta^{2} T\left[a_{0}, v_{0}\right]\right]\right\},
$$

which is a first-order differential equation. Notice that there is no dependence on the free parameter $\tilde{\beta}$. This is not surprising if one takes into account that $\tilde{\beta}$ is the coefficient of a term proportional to $\square R$ in the trace [see Eqs. (28) and (29)] and that for the classical background $R_{0}=4 \Lambda$ is a constant (where $R_{0}$ is the Ricci scalar for the classical background).

Let us now compute the nonlocal term $T\left[a_{0}, v_{0}\right]$ in the previous equation. After substitution of $a_{0}$ into Eq. (44), the state-independent contribution of this term reads

$$
T_{1}\left[a_{0} ; \eta\right)=-\frac{3}{2 \eta^{4}}+\frac{1}{\eta_{i}^{2} \eta^{2}}+\frac{4}{\eta_{i}^{3} \eta}-\frac{7}{2 \eta_{i}^{4}},
$$

which is independent of the arbitrary renormalization scale $\mu$. The latter is again because when substituting the local part of $\kappa_{1}\left[f ; \eta^{\prime}\right)$ into Eq. (44), one obtains an integrand proportional to $\square R_{0}=0$; this point could also have been anticipated from the fact that the term proportional to $\ln \mu$ should have the same form as the term proportional to $\tilde{\beta}$, which has been found to vanish above. ${ }^{4}$

Substituting $a_{0}$ and $v_{0}$ into Eq. (45), we get the following result for the state-dependent part of $T\left[a_{0}, v_{0} ; \eta\right)$ :

$$
\begin{aligned}
T_{2}\left[a_{0}, v_{0} ; \eta\right)= & \int_{\eta_{i}}^{\eta} d \eta^{\prime} \int_{-\infty}^{\eta_{i}} \frac{d \eta^{\prime \prime}}{\eta^{\prime}-\eta^{\prime \prime}} \\
& \times\left\{\frac{2}{\eta^{\prime 3}} v_{0}\left(\eta^{\prime \prime}\right)-\frac{2}{\eta^{\prime 2}} \dot{v}_{0}\left(\eta^{\prime \prime}\right)-\frac{1}{\eta^{\prime}} \ddot{v}_{0}\left(\eta^{\prime \prime}\right)\right\} \\
& +\frac{1}{\eta_{i}^{2}} \kappa\left[v_{0} ; \eta_{i}\right)+\frac{1}{\eta_{i}} \kappa\left[\dot{v}_{0} ; \eta_{i}\right) \\
& +\int_{-\infty}^{\eta_{i}} d \eta^{\prime} v_{0}\left(\eta^{\prime}\right) \kappa\left[\dot{v}_{0} ; \eta^{\prime}\right) .
\end{aligned}
$$

The conditions (50) guarantee that the dependence on $\mu$ of the last three terms cancels out. The integral over $\eta^{\prime}$ in the first term can be easily computed. Finally, adding up the two contributions $T_{1}$ and $T_{2}$ we get

$$
\begin{aligned}
T\left[a_{0}, v_{0} ; \eta\right)= & -\frac{3}{2 \eta^{4}}+\frac{A_{\Psi}}{\eta^{2}}+B_{\Psi} \\
& +\int_{-\infty}^{\eta_{i}} d \eta^{\prime} \ln \left[\frac{\eta_{i}\left(\eta-\eta^{\prime}\right)}{\eta\left(\eta_{i}-\eta^{\prime}\right)}\right] \\
& \times\left[\frac{2}{\eta^{\prime 3}} v_{0}\left(\eta^{\prime}\right)-\frac{2}{\eta^{\prime 2}} \dot{v}_{0}\left(\eta^{\prime}\right)-\frac{1}{\eta^{\prime}} \ddot{v}_{0}\left(\eta^{\prime}\right)\right]
\end{aligned}
$$

where

\footnotetext{
${ }^{4}$ Note that the results obtained in Ref. [27] in a similar context exhibit a nonvanishing dependence on $\mu$. This should not be the case for the reasons given here.
}

$$
A_{\Psi}=\frac{1}{\eta_{i}^{2}}+\int_{-\infty}^{\eta_{i}} d \eta^{\prime} \frac{v_{0}\left(\eta^{\prime}\right)}{\eta^{\prime}}
$$

and

$$
\begin{aligned}
B_{\Psi}= & \frac{1}{2 \eta_{i}^{4}}-\frac{1}{\eta_{i}^{2}} \int_{-\infty}^{\eta_{i}} d \eta^{\prime} \frac{v_{0}\left(\eta^{\prime}\right)}{\eta^{\prime}}+\frac{1}{\eta_{i}^{2}} \kappa\left[v_{0} ; \eta_{i}\right) \\
& +\frac{1}{\eta_{i}} \kappa\left[\dot{v}_{0} ; \eta_{i}\right)+\int_{-\infty}^{\eta_{i}} d \eta^{\prime} v_{0}\left(\eta^{\prime}\right) \kappa\left[\dot{v}_{0} ; \eta^{\prime}\right) .
\end{aligned}
$$

We have already pointed out that the first-order equation (51) includes neither the free parameter $\tilde{\beta}$ nor the arbitrary renormalization mass scale $\mu$. Moreover, it does not depend on $\alpha$ either since $A_{\mu \nu}=0$ for a conformally flat spacetime. This means that the semiclassical Friedmann equation (48) is fully predictive to this first perturbative order for the particular case of a de Sitter background that we are considering.

\section{B. The solution}

Equation (51) for the function $a_{1}(\eta)$ is a first-order, linear differential equation, which can be easily solved in an explicit form. The general solution of a differential equation of the type $y^{\prime}(x)=-(2 / x) y+f(x)$ is $y(x)=$ $k / x^{2}+\left(1 / x^{2}\right) \int d x x^{2} f(x)$, where $k$ is an arbitrary integration constant. Therefore, the solution of Eq. (51) is

$$
\begin{aligned}
a_{1}(\eta)= & \frac{k}{H^{2} \eta^{2}}+\left(\frac{1}{720 \pi}-\frac{3 \nu^{2}}{2 \pi}\right) \frac{1}{H \eta} \\
& -\frac{3 \nu^{2}}{\pi H}\left[\frac{A_{\Psi}}{3} \eta+\frac{B_{\Psi}}{5} \eta^{3}+\Gamma_{\Psi}(\eta)\right],
\end{aligned}
$$

where

$$
\begin{aligned}
\Gamma_{\Psi}(\eta)= & \int_{-\infty}^{\eta_{i}} d \eta^{\prime} \gamma\left(\eta, \eta^{\prime}\right)\left[\frac{2}{\eta^{\prime 3}} v_{0}\left(\eta^{\prime}\right)-\frac{2}{\eta^{\prime 2}} \dot{v}_{0}\left(\eta^{\prime}\right)\right. \\
& \left.-\frac{1}{\eta^{\prime}} \ddot{v}_{0}\left(\eta^{\prime}\right)\right]
\end{aligned}
$$

with

$$
\begin{aligned}
\gamma\left(\eta, \eta^{\prime}\right)= & \frac{1}{5 \eta^{2}}\left\{\eta^{5} \ln \left[\frac{\eta_{i}\left(\eta-\eta^{\prime}\right)}{\eta\left(\eta_{i}-\eta^{\prime}\right)}\right]\right. \\
& -\eta^{\prime 5}\left[\frac{1}{4}\left(\frac{\eta}{\eta^{\prime}}\right)^{4}+\frac{1}{3}\left(\frac{\eta}{\eta^{\prime}}\right)^{3}+\frac{1}{2}\left(\frac{\eta}{\eta^{\prime}}\right)^{2}+\frac{\eta}{\eta^{\prime}}\right. \\
& \left.\left.+\ln \left(1-\frac{\eta}{\eta^{\prime}}\right)\right]\right\}
\end{aligned}
$$

The condition (7), which $v_{0}(\eta)$ must also satisfy, ensures that the integral in Eq. (58) is finite in its lower limit. As for the upper limit, all the possible divergences in the integrand cancel out (moreover, even if they did not cancel out, they would give a finite contribution when integrated since they are logarithmic). Thus, $\Gamma_{\Psi}(\eta)$ is finite and, furthermore, it vanishes at future infinity, i.e., when $\eta \rightarrow 0^{-}$. 
The solutions associated with the different possible values of the arbitrary integration constant $k$ are related to each other by time translations: $a\left(\eta+\left(l_{p} H\right)^{2} \Delta\right)=$ $-1 / H \eta+\left(l_{p} H\right)^{2}\left[a_{1}(\eta)+\Delta / H \eta^{2}\right]+O\left(\left(l_{p} H\right)^{4}\right)$. For the particular case $k=0$, and provided that $H^{-1} \gg l_{p}$, the first-order term in the expansion (49) is much smaller than the zeroth-order term for all times $\eta_{i}<\eta<0$, and one expects that the higher-order corrections can be neglected [since they are suppressed by an additional power of $\left.\left(l_{p} H\right)^{2}\right]$ and the solution $a(\eta)$ can be approximated by the first-order perturbative expansion. This approximation is no longer suitable for solutions (initial states) associated with other values of $k$, but we already know that they are just time translations of the solutions with $k=0$. Therefore, we can conclude that for reasonable states, namely, those which do not involve Planckian scales (and up to time translations) the scale factor after the initial time $\eta_{i}$ is given by

$$
\begin{aligned}
a(\eta)= & -\frac{1}{\tilde{H} \eta}-\left(l_{p} H\right)^{2} \frac{3 \nu^{2}}{\pi H}\left[\frac{A_{\Psi}}{3} \eta+\frac{B_{\Psi}}{5} \eta^{3}+\Gamma_{\Psi}(\eta)\right] \\
& +O\left(\left(l_{p} H\right)^{4}\right),
\end{aligned}
$$

where the modified Hubble constant is

$$
\tilde{H}=H\left[1+\left(l_{p} H\right)^{2}\left(\frac{1}{720 \pi}-\frac{3 \nu^{2}}{2 \pi}\right)\right] .
$$

The result in Eq. (60) is valid for all times (after the initial time). It is the sum of a corrected de Sitter solution plus terms that vanish at future infinity $\eta \rightarrow 0^{-}$. The corrected de Sitter term dominates at late times, regardless of the initial state. Therefore, we conclude that de Sitter spacetime is stable under spatially isotropic perturbations in semiclassical gravity. Note that for extremely excited states (highly squeezed), i.e., for sufficiently large values of $A_{\Psi}$, $B_{\Psi}$, and $\Gamma_{\Psi}\left(\eta_{i}\right)$, the perturbative expansion in Eq. (60) would break down at early times. It may still be possible to solve the semiclassical equation consistently by using some order-reduction method and solving the resulting backreaction equation exactly rather than considering a perturbative solution. However, although this would happen well before the energy density of the quantum field becomes Planckian, and a low-energy effective field theory approach should still be possible, the quantum fluctuations of the metric around the mean background geometry can then become important even for scales larger than the Planck length and the usual mean field approximation where the fluctuations are treated perturbatively, may no longer be adequate. In any case, our explicit perturbative solution will at least signal whether that can happen for a given initial state.

The semiclassical shift of the Hubble constant does not have a definite sign; it depends on the coupling between the quantum field and the curvature. For instance, while the shift is positive for the conformal coupling case $(\nu=0)$, when $\nu=-1 / 6$, which is expected to mimic the effect of gravitons, this shift is negative, implying a small timeindependent screening of the cosmological constant. The deviation from the conformal coupling, $\nu$, is what makes the Hamiltonian (5) time dependent through the term involving the scale factor. This is what allows us to prepare a wide range of initial states by evolution of the in-vacuum with different scale factors $a_{\Psi}(\eta)$. In fact, the effect of this time dependence of the Hamiltonian can be interpreted as particle creation. Since the term proportional to $\nu^{2}$ in Eq. (61) is negative, one can say that at late times the effect of the created particles is to slow down the de Sitter expansion by a small amount, whereas the other vacuum polarization term, already present in the conformal case, has the opposite effect.

A particularly interesting initial state is the BunchDavies vacuum $\left|\Psi_{\mathrm{BD}}\left(\eta_{i}\right)\right\rangle$, which is obtained by evolution of the in-vacuum from $-\infty$ to $\eta_{i}$ according to the scale factor of de Sitter spacetime or, in other words, with $v_{0}(\eta)=2 / \eta^{2}$. In this case, one can check that $A_{\Psi}=$ $B_{\Psi}=\Gamma_{\Psi}(\eta)=0$, so the solution (60) is just the de Sitter solution with a semiclassically modified Hubble parameter. Therefore, the Bunch-Davies vacuum together with de Sitter spacetime with the semiclassically modified Hubble constant constitutes a self-consistent solution of the semiclassical Friedmann equation, to which other perturbed solutions tend at late times. This self-consistent solution corresponds to the solution close to the classical one found in Ref. [37]. On the other hand, the second solution found in Ref. [37] for the massless case (when $\nu^{2}<1 / 1080$ ) exhibits characteristic scales of the order of the Planck length and lies beyond the domain of validity of a low-energy effective field theory approach; it was automatically excluded in our perturbative treatment in powers of $\left(l_{p} H\right)^{2}$.

\section{DISCUSSION}

In this paper we have computed the one-loop vacuum polarization for massless nonconformal fields in a general spatially flat RW background. This has then been applied to studying the evolution of spatially isotropic perturbations around de Sitter spacetime when the backreaction due to a massless nonconformal field is self-consistently included, which corresponds to solving the equations of semiclassical gravity for this case. There is a selfconsistent solution, associated with the Bunch-Davies vacuum for the quantum fields, with an effective cosmological constant slightly shifted from its classical value due to the vacuum polarization effects. Furthermore, we have found that this solution is stable under spatially isotropic perturbations since the perturbed solutions tend to it at late times. It should be stressed that our results are independent of the particular value of the renormalization parameters $\alpha$ and $\beta$, and therefore fully predictive (at the first order in $l_{p}^{2}$ at which we are working). It should also be pointed out that 
our results seem to be at variance with those of Ref. [27]. In this respect, it is important to keep in mind that one should consider the stability of the self-consistent solution with the shifted effective cosmological constant rather than that of the classical solution obtained in the absence of vacuum polarization effects. Moreover, when comparing the perturbed solution to the self-consistent de Sitter "attractor" it is important to properly take into account any relative conformal-time translation since that can give rise to a spurious growth in time of their ratio. This can be clearly illustrated by comparing two copies of the same selfconsistent de Sitter solution with a relative conformaltime translation. Finally, as already emphasized in the Introduction, a complete analysis of the backreaction problem in de Sitter spacetime and its stability should take into account the effect of the quantum metric fluctuations as well. Including the metric fluctuations is certainly a crucial aspect, and some steps in that direction are briefly discussed below. However, given the complexity of a completely satisfactory treatment involving the quantized metric perturbations, it is important to make sure that there are no significant effects even when they are not taken into account, especially because such effects have actually been suggested by a number of studies in the literature.

We were able to obtain explicit analytic results by using two approximations. First, we considered a perturbative expansion in the parameter $\nu$, which characterizes the deviation of the curvature-coupling parameter from the conformal case, and truncated the expansion to quadratic order. Hence, our effective action is exact through order $\nu^{2}$. Second, we introduced a perturbative expansion in powers of $\left(l_{p} H\right)^{2}$. Its purpose was to obtain a fairly accurate description for phenomena involving length scales much larger than the Planck length while discarding spurious solutions involving Planckian scales, where the effective field theory approach that we have been using breaks down. Truncating such a perturbative expansion for the solutions (rather than doing so at the level of the equation of motion and then solving it exactly) can sometimes miss the right long-time behavior. However, as we discussed in Sec. V, this should not be the case for the situation considered here.

There are a number of natural extensions or generalizations of our work in this paper. First, one could consider the other possibility for having weakly nonconformal fields, namely, the case of fields with conformal coupling but with a small nonvanishing mass such that $m^{2} \ll H^{2}$. In that case one should be able to proceed analogously to what we did here by computing the effective action perturbatively in $m^{2}$ through order $m^{4}$ and then solving the equation of motion through order $\left(l_{p} H\right)^{2}$. Second, the case of strongly nonconformal fields with a large mass $M^{2} \gg H^{2}$ could be explicitly calculated using an adiabatic (or WKB) expansion [65]. The effective action in that case could be written as a local expansion of positive powers of curvature invariants suppressed by the corresponding power of $M^{2}$, a form which can be anticipated from local effective field theory arguments based on power counting and taking into account the relevant symmetries (diffeomorphism invariance in this case). On the other hand, the case of strong nonconformal coupling to the curvature could be treated by introducing a field-dependent conformal transformation relating the original Jordan frame to the Einstein frame, ${ }^{5}$ where the curvature scalar does not couple to the scalar field [67]. The case of massive fields for both small and large masses, which correspond, respectively, to the weakly and strongly nonconformal regimes, is analyzed in detail in Ref. [68]. The effective action calculated perturbatively for the small mass case has the same form as that obtained in Ref. [69] for one-loop graviton effects. Therefore, one can compare the result for solutions of the backreaction equation for the small mass case in Ref. [68] to that in Ref. [69].

Third, even though the construction based on Eq. (6) can generate a fairly wide family of squeezed Gaussian states by considering a sufficiently general form of the auxiliary scale factor $a_{\Psi}(\eta)$, other approaches are needed in order to deal with all possible Gaussian states or even non-Gaussian ones. One possibility is to make use of the method developed in Ref. [40] for a fixed background spacetime, which is based on the construction of fourth-order adiabatic vacuum states (one can show that the classes of states that we have considered here are compatible with their approach and encompassed by the general class of states it can deal with). However, even if we restrict ourselves to the subclass of initial states generated by Eq. (6), the procedure can be straightforwardly generalized to states with nonvanishing expectation values of the field or its canonically conjugate momentum. Since we are considering Gaussian states, this can be done by decomposing the field as a sum of a classical part which characterizes the evolution of the expectation value and satisfies the classical equation of motion plus a field with vanishing expectation value whose initial state can be generated by Eq. (6). This implies that when solving the backreaction equation in powers of $\left(l_{p} H\right)^{2}$ one first needs to find the self-consistent solution for the background scale factor and the classical configuration of the field (corresponding to the evolution of its expectation value), and then solve for the perturbation of the scale factor due to the vacuum polarization effect of the quantum fluctuations evolving on that background geometry.

\footnotetext{
${ }^{5}$ Any physical predictions derived in the Jordan and Einstein frames should be equivalent, at least at the classical level. In the quantum mechanical case one still expects such an equivalence for small perturbations of the metric and the scalar field around their mean values [66]. In that case, however, it may be necessary to treat the perturbations of the metric and the scalar field on an equal footing, and the metric perturbations may need to be quantized.
} 
Throughout the paper we have considered spatially isotropic perturbations, i.e., RW geometries and quantum states compatible with their symmetries (spatial homogeneity and isotropy). It would be interesting to study the stability of de Sitter spacetime with respect to general inhomogeneous and anisotropic perturbations. In that case one has nontrivial results even for a massless and conformally coupled field. The underlying reason is that when considering inhomogeneous and anisotropic metric perturbations, the perturbed geometry is no longer conformally flat. Whereas the stability of Minkowski spacetime with respect to general linear perturbations has been studied for arbitrary masses and curvature couplings (see Refs. [33,70] and references therein), such results do not exist for RW backgrounds. In order to analyze the dynamics of inhomogeneous and anisotropic perturbations around a spatially flat RW background one can make use of the effective action and the semiclassical Einstein equation for general linear perturbations around a spatially flat RW background obtained in Ref. [43] for a massless and conformally coupled field (see also Ref. [55] for a more compact form). Unfortunately this linearized semiclassical equation is a complicated integro-differential equation and its solutions have not been studied in detail so far ${ }^{6}$; see, however, Ref. [71] for recent work in this direction.

We have studied the backreaction of the quantum fields on the dynamics of the spacetime geometry within the framework of semiclassical gravity, which can be understood as a mean field approximation where the mean gravitational field is described by a classical metric whereas its quantum fluctuations are not considered. In order to study the quantum fluctuations of the gravitational field one can consider the metric perturbations around a background geometry corresponding to the semiclassical gravity solution and quantize them within a low-energy effective field theory approach to quantum gravity $[28,29,72,73]$. So far this approach has been mostly applied to weak-field problems [30], but it seems particularly interesting to extend its application to strong-field situations involving black holes and cosmological spacetimes $[74,75]$. The stochastic gravity formalism $[50,51]$ can be a useful tool in this respect since one can prove its equivalence to a quantum treatment of the metric perturbations if graviton loops are neglected, which can be formally justified in a large $N$ expansion for a large number of matter fields [76]. A central object in this formalism is the symmetrized connected two-point function of the stress-tensor operator for the quantum matter fields, which determines

\footnotetext{
${ }^{6}$ For perturbations around Minkowski it is relatively easy to solve the integro-differential equation because it can be transformed into a purely algebraic equation by Fourier transforming with respect to not only the spatial coordinates but also the time coordinate. By contrast, that is not possible in the RW case due to the time dependence of the scale factor, and the nonlocality in time cannot be eliminated.
}

the metric fluctuations induced by the quantum fluctuations of the matter fields. Such an object has been computed for a massless minimally coupled field evolving in a de Sitter background spacetime and the fluctuations of the stress tensor were found to be comparable to its expectation value [77]. Therefore, studying in detail the quantum fluctuations of the metric in this context constitutes a natural extension of our work worth pursuing. The results obtained here would still be relevant in that case because they provide the right background around which the metric should be perturbed and quantized.

We close this section with a brief discussion of the relationship between our results and the linearization instability for metric perturbations around de Sitter spacetime coupled to a scalar field found in Ref. [78], where it was concluded that it is only consistent to consider de Sitter invariant states for the quantum field. This conclusion does not directly affect our analysis because we did not consider fluctuations of the metric and studied only the dynamics of the mean geometry, which couples to the expectation value of the stress-tensor operator of the matter field. The expectation value of the stress tensor for the class of states that we have considered in this paper, which are spatially homogenous and isotropic, automatically satisfies the linearization stability constraint given by Eq. (44) in Ref. [78]. It is when considering the quantum fluctuations of the metric that the linearization stability condition imposes additional restrictions on the state of the matter field because in that case the condition must be imposed on the $n$-point correlation functions of the stress tensor as well, and this implies that the state of the field must be de Sitter invariant.

\section{ACKNOWLEDGMENTS}

We are grateful to Daniel Arteaga, Diego Blas, Joan Antoni Cabrer, Domènec Espriu, Jaume Garriga and Emil Mottola for interesting discussions. This work has been partly supported by the Research Projects No. MEC FPA-2004-04582, No. MEC FPA-2007-66665-C02-02 and No. DURSI 2005SGR00082. A. R. is supported by LDRD funds from Los Alamos National Laboratory.

\section{APPENDIX A: SEMICLASSICAL EINSTEIN EQUATION}

In this Appendix we briefly review the semiclassical Einstein equation and its derivation by functional techniques. When neglecting the effects of graviton loops, the backreaction of quantum matter fields on the mean gravitational field is described by the semiclassical Einstein equation, which can be written as

$$
G_{a b}[g]+\Lambda g_{a b}+\alpha A_{a b}[g]+\beta B_{a b}[g]=\kappa\left\langle\hat{T}_{a b}[g]\right\rangle_{\mathrm{ren}},
$$

where $G_{a b}$ is the Einstein tensor associated with some 
globally hyperbolic spacetime with metric $g_{a b},\left\langle\hat{T}_{a b}[g]\right\rangle_{\text {ren }}$ is the suitably renormalized expectation value of the stresstensor operator corresponding to the scalar field operator $\hat{\phi}[g]$, and $\alpha, \beta, \Lambda$, and $\kappa$ are renormalized parameters evaluated at the same renormalization scale as $\left\langle\hat{T}_{a b}[g]\right\rangle_{\text {ren }}$. The tensors $A_{a b}$ and $B_{a b}$ are obtained by functionally differentiating with respect to the metric terms corresponding to the Lagrangian densities $C^{a b c d} C_{a b c d}$ and $R^{2}$ in the gravitational action,

$$
\begin{gathered}
A^{a b}=\frac{1}{\sqrt{-g}} \frac{\delta}{\delta g_{a b}} \int d^{4} x \sqrt{-g} C_{c d e f} C^{c d e f}, \\
B^{a b}=\frac{1}{\sqrt{-g}} \frac{\delta}{\delta g_{a b}} \int d^{4} x \sqrt{-g} R^{2},
\end{gathered}
$$

where $C_{a b c d}$ is the Weyl tensor and $R$ the Ricci curvature scalar. These Lagrangian densities $C_{a b c d} C^{a b c d}$ and $R^{2}$ are related to the counterterms introduced in the bare gravitational action needed to renormalize the ultraviolet divergences arising in the expectation value of the stress tensor. Note that from their definitions the tensors (A2) and (A3) are divergenceless: $\nabla^{a} A_{a b}=0=\nabla^{a} B_{a b}$.

Let us see how the semiclassical Einstein equation can be derived by functional methods. To compute the expectation value on the right-hand side of Eq. (A1) we can use the closed time path (CTP) or "in-in" functional formalism [43,74,79-83]. Let us foliate the assumed globally hyperbolic spacetime with $t=$ const spacelike hypersurfaces $\Sigma_{t}$, and denote the initial and final times by $t_{i}$ and $t_{f}$, respectively. In the CTP formalism we introduce two copies of the metric and the field, $\left(g_{a b}^{+}, g_{a b}^{-}\right)$and $\left(\phi^{+}, \phi^{-}\right)$, which will coincide at the final time: $g_{a b}^{+}\left(t_{f}\right)=g_{a b}^{-}\left(t_{f}\right)$ and $\phi^{+}\left(t_{f}\right)=\phi^{-}\left(t_{f}\right)$. Let $\rho_{i}\left[\phi^{+}\left(t_{i}\right), \phi^{-}\left(t_{i}\right)\right]$ be the matrix element of the density operator describing the initial state of the scalar field. The Feynman-Vernon influence action [84,85], $S_{\mathrm{IF}}\left[g^{+}, g^{-}\right]$, which describes the effect of the matter field on the gravitational field, is defined as the following path integral over two copies of the scalar field:

$$
\begin{aligned}
e^{i S_{\mathrm{IF}}\left[g^{+}, g^{-}\right]}= & \int \mathcal{D} \phi^{+} \mathcal{D} \phi^{-} \rho_{i}\left[\phi^{+}\left(t_{i}\right), \phi^{-}\left(t_{i}\right)\right] \\
& \times \delta\left[\phi^{+}\left(t_{f}\right)-\phi^{-}\left(t_{f}\right)\right] e^{i\left(S_{m}\left[g^{+}, \phi^{+}\right]-S_{m}\left[g^{-}, \phi^{-}\right]\right.},
\end{aligned}
$$

where $S_{m}[g, \phi]$ is the action for the scalar field in the spacetime described by the metric $g_{a b}$. Neglecting graviton loops, the CTP effective action for the gravitational field is then

$$
\Gamma\left[g^{+}, g^{-}\right]=S_{g}\left[g^{+}\right]-S_{g}\left[g^{-}\right]+S_{\mathrm{IF}}\left[g^{+}, g^{-}\right],
$$

where $S_{g}\left[g^{ \pm}\right]$is the bare gravitational action. $\Gamma\left[g^{+}, g^{-}\right]$is the effective action for the mean gravitational field coupled to the quantum scalar field. $S_{\mathrm{IF}}\left[g^{+}, g^{-}\right]$has ultraviolet divergences which can be renormalized by using a suitable regularization procedure and by adding the aforementioned counterterms to the bare gravitational action $S_{g}\left[g^{ \pm}\right]$. More specifically, one starts with a regularized gravitational action in Eq. (A5) which includes the bare parameters $\kappa_{B}, \Lambda_{B}, \alpha_{B}, \beta_{B}$; at the end of the calculation one takes the regularization parameter to its physical value and the divergences are absorbed into the bare parameters which acquire their dressed physical values. The renormalized effective action can then be written as

$$
\Gamma\left[g^{+}, g^{-}\right]=S_{g}^{\mathrm{ren}}\left[g^{+}\right]-S_{g}^{\mathrm{ren}}\left[g^{-}\right]+S_{\mathrm{IF}}^{\mathrm{ren}}\left[g^{+}, g^{-}\right],
$$

where the superscript means that these terms have been already renormalized and are finite.

Since the classical stress tensor of the matter field is defined as

$$
T^{a b}=\frac{2}{\sqrt{-g}} \frac{\delta S_{m}}{\delta g_{a b}},
$$

one can see from the definition of the influence action in Eq. (A4) that the expectation value of the stress tensor in the given quantum state of the field is given by

$$
\left\langle\hat{T}^{a b}[g]\right\rangle_{\mathrm{ren}}=\left.\frac{2}{\sqrt{-g}} \frac{\delta S_{\mathrm{IF}}^{\mathrm{ren}}\left[g^{+}, g^{-}\right]}{\delta g_{a b}^{+}}\right|_{g^{+}=g^{-}=g},
$$

where the renormalized value of the influence action has been used. Finally, the semiclassical Einstein equations (A1) can be derived by functional derivation of the effective gravitational action, $\Gamma\left[g^{+}, g^{-}\right]$, with respect to $g_{a b}^{+}$and then taking $g_{a b}^{+}=g_{a b}^{-}=g_{a b}$ :

$$
\left.\frac{\delta \Gamma\left[g^{+}, g^{-}\right]}{\delta g_{a b}^{+}}\right|_{g^{+}=g^{-}=g}=0 .
$$

Notice that doubling the number of fields, the "plus" field, which evolves forward in time, and "minus" field, which evolves backward in time, is what allows us to obtain an expectation value from the above functional derivative in the CTP formalism, rather than a transition element as in the ordinary "in-out" effective action method. This can be clearly seen from the following alternative representation of the influence action:

$$
\begin{aligned}
e^{i S_{\mathrm{IF}}\left[g^{+}, g^{-}\right]} & =\operatorname{tr}\left[\hat{U}_{g^{+}}\left(t_{f}, t_{i}\right) \hat{\rho}_{i} \hat{U}_{g^{-}}^{\dagger}\left(t_{f}, t_{i}\right)\right] \\
& =\left\langle\Psi_{i}\left|\hat{U}_{g^{-}}\left(t_{i}, t_{f}\right) \hat{U}_{g^{+}}\left(t_{f}, t_{i}\right)\right| \Psi_{i}\right\rangle,
\end{aligned}
$$

where the last equality holds for a pure initial state of the field $\hat{\rho}_{i}=\left|\Psi_{i}\right\rangle\left\langle\Psi_{i}\right|$ and $\hat{U}_{g^{ \pm}}$is the unitary time evolution operator for a field $\hat{\phi}$ propagating in a spacetime with metric $g_{a b}^{ \pm}$:

$$
\hat{U}_{g^{ \pm}}\left(t_{f}, t_{i}\right)=T \exp \left(-i \int_{t_{i}}^{t_{f}} d t \hat{H}_{m}\left[g^{ \pm}, \hat{\phi}, \hat{\pi}\right]\right),
$$

where $T$ denotes time ordering and $\hat{H}_{m}[g, \hat{\phi}, \hat{\pi}]$ is the Hamiltonian of the scalar field obtained by considering 
the spacetime foliation $\left\{\Sigma_{t}\right\}$ and introducing the corresponding $3+1$ decomposition.

In this paper we use dimensional regularization, which means that the regularization parameter is $n-4$, where $n$ is the number of spacetime dimensions. In that case the square of the Weyl tensor in the Lagrangian density of Eq. (A2) must be substituted by $\frac{2}{3}\left(R_{a b c d} R^{\mu \nu \rho \sigma}-R_{a b} R^{a b}\right)$, where $R_{a b c d}$ and $R_{a b}$ are the Riemann and Ricci tensors. Such a Lagrangian density reduces to the square of the Weyl tensor in $n=4$ when the Gauss-Bonnet theorem is taken into account.

We also consider a massless scalar field throughout. Using dimensional regularization the counterterms for a massless scalar field can be read from the divergent parts in the effective action computed using the DeWitt-Schwinger expansion of the Feynman propagator in the coincidence limit; see Eq. (6.44) in Ref. [31]. It should be remarked that the DeWitt-Schwinger expansion is defined for massive fields and it is ill defined in the massless limit. However, it can still be used to extract the divergent terms. There are three divergent terms in this expansion; the first and second terms have coefficients related to a constant and to the Ricci scalar, respectively. These two terms vanish in the massless limit. The third term has a coefficient which is quadratic in the curvature, and in this case it is convenient to keep the mass as an infrared regulator which can be removed at the end of the calculation by redefining the renormalization scale. This term leads to the following counterterm for the gravitational action:

$$
\begin{aligned}
S_{g}^{c}[g ; \mu]= & \left(\alpha(\mu)+\frac{\mu^{n-4}}{2880 \pi^{2}(n-4)}\right) \\
& \times \int d^{n} x \sqrt{-g}\left(R_{a b c d} R^{a b c d}-R_{a b} R^{a b}\right) \\
& +\left(\beta(\mu)+\frac{\nu^{2} \mu^{n-4}}{32 \pi^{2}(n-4)}\right) \int d^{n} x \sqrt{-g} R^{2},
\end{aligned}
$$

where $\alpha(\mu)$ and $\beta(\mu)$ are the renormalized dimensionless parameters which appear in Eq. (A1), and $\mu$ is a parameter with dimensions of mass which ensures that the action has the correct dimensions even when $n \neq 4$ and plays the role of the renormalization scale in dimensional regularization. The bare parameters $\alpha_{B}$ and $\beta_{B}$, which correspond to the whole factor multiplying the first and second integrals, respectively, should be independent of the renormalization scale $\mu$. Therefore, under a change of the renormalization scale $\mu \rightarrow \mu^{\prime}$ [and neglecting terms with positive powers of $(n-4)$, which vanish when $n \rightarrow 4]$ the renormalized parameters change as follows: $\alpha\left(\mu^{\prime}\right)=\alpha(\mu)-$ $\left(2880 \pi^{2}\right)^{-1} \ln \left(\mu^{\prime} / \mu\right)$ and $\beta\left(\mu^{\prime}\right)=\beta(\mu)-\left(\nu^{2} / 32 \pi^{2}\right) \times$ $\ln \left(\mu^{\prime} / \mu\right)$.

Note that as seen from Eq. (23), the $R^{2}$ counterterm in the action can be easily inferred from the $(n-4)^{-1}$ divergence in Eq. (20), but it is not so obvious how to come up with the other counterterm (with the Riemann square) because no other divergence is present. That is because the first term in Eq. (19), which would be the entire contribution for a conformal field, is finite in the particular case of a conformally flat metric. However, a slight departure from conformal flatness (e.g., by considering inhomogeneous perturbations around the flat space geometry) renders the first term in Eq. (19) divergent and such a divergence is accounted for by the above general DeWittSchwinger expansion $[86,87]$. Hence, even though the first term in Eq. (19) would still be independent of $a$ when regulated with dimensional regularization, the counterterms that cancel the divergences in the limit $n \rightarrow 4$ would bring the $a$ dependence into the problem.

\section{APPENDIX B: REGULAR INITIAL STATES}

As has been pointed out in Sec. IV B, the expectation value of the trace of the stress tensor diverges at the initial time unless we choose an appropriate vacuum state for the quantum field. Such an appropriate vacuum state is obtained when the scale factors before and after the initial time match with continuity up to the fourth derivative, as given by Eq. (40). In this Appendix we will examine this point in more detail. First, closely following Ref. [40] we will derive the condition that any homogeneous and isotropic vacuum must satisfy in order for the trace to be finite at the initial time. This is a well-known result: the vacuum must be fourth-order adiabatic (see, for instance, $[40,88]$ ). Next, we will specialize this condition to the class of vacuum states that has been considered in this paper, and show that it is equivalent to the "smoothness" condition (40). In other words, among all the vacuum states considered in this paper Eq. (40) selects those that are fourthorder adiabatic.

Let us first define what we mean by a general homogeneous and isotropic vacuum. Setting the variation of the classical action (4) with respect to the scalar field $\phi$ equal to zero yields the dynamical equation for the scalar field, i.e., the Klein-Gordon equation

$$
\left(\eta^{\mu \nu} \partial_{\mu} \partial_{\nu}-M^{2}\right) \phi=0,
$$

where the time-dependent mass $M(\eta)$ is defined as $M^{2}=$ $\nu a^{2} R$. This equation admits a complete set of orthonormal solutions of the form

$$
u_{\vec{k}}(\eta, \vec{x})=f_{k}(\eta) \frac{1}{(2 \pi)^{3 / 2}} e^{i \vec{k} \cdot \vec{x}},
$$

where $\vec{k} \in \mathbb{R}^{3}$, and $k=|\vec{k}|$. The Klein-Gordon equation (B1) implies that the mode functions $f_{k}$ satisfy the equation of a harmonic oscillator with a timedependent frequency:

$$
\ddot{f}_{k}+\Omega_{k}^{2} f_{k}=0,
$$

where the time-dependent frequency $\Omega_{k}(\eta)$ is given by 
$\Omega_{k}^{2}=k^{2}+M^{2}$. In order for the set $\left\{u_{\vec{k}}\right\}$ to be orthonormal, the mode functions must also satisfy the Wronskian condition

$$
\dot{f}_{k} f_{k}^{*}-f_{k} \dot{f}_{k}^{*}=-i .
$$

The general operator-valued solution of the Klein-Gordon equation (B1) can be written as a linear combination of the orthonormal solutions:

$$
\hat{\phi}=\int d^{3} k\left(\hat{a}_{\vec{k}} u_{\vec{k}}+\hat{a}_{\vec{k}}^{\dagger} u_{\vec{k}}^{*}\right),
$$

where $\hat{a}_{\vec{k}}$ and $\hat{a}_{\vec{k}}^{\dagger}$ are the annihilation and creation operators, respectively. The vacuum state $|0\rangle$ is defined as the state annihilated by all the annihilation operators: $a_{\vec{k}}|0\rangle=$ 0 for all $\vec{k}$. Because of the particular spatial dependence of the orthonormal solutions $u_{\vec{k}}$, this vacuum is invariant under spatial translations and rotations, i.e., it is homogeneous and isotropic. Note that the vacuum is not fully specified until we give initial conditions for the solutions of the mode equation (B3), which must also be consistent with the Wronskian condition (B4). Different choices of initial conditions give rise to different sets of mode functions, and thus to different homogeneous and isotropic vacua.

The bare expectation value of the trace of the stress tensor in a general homogeneous and isotropic vacuum can be written as a sum over all the modes associated with it (see, for instance, Ref. [88]):

$$
\begin{aligned}
\left\langle T_{\mu}^{\mu}\right\rangle= & -\frac{6 \nu}{2 \pi^{2} a^{4}} \int_{0}^{\infty} d k k^{2}\left[\dot{h}\left|f_{k}\right|^{2}+2 h\left|f_{k}\right| \frac{d\left|f_{k}\right|}{d \eta}\right. \\
& \left.-\left|\dot{f}_{k}\right|^{2}+\Omega_{k}^{2}\left|f_{k}\right|^{2}\right],
\end{aligned}
$$

where $h \equiv \dot{a} / a$. This equation is obtained by substituting Eq. (B5) into the definition of the stress-tensor operator in terms of the rescaled field $\hat{\phi}$, and making use of the standard commutation relations among the creation and annihilation operators. In order to renormalize this expectation value, let us introduce the WKB mode functions. The most general mode function satisfying the Wronskian condition (B4) can be written as

$$
f_{k}(\eta)=\frac{1}{\sqrt{2 W_{k}}} \exp \left[-i \int_{\eta_{i}}^{\eta} d \eta^{\prime} W_{k}\left(\eta^{\prime}\right)\right],
$$

with real, positive $W_{k}$. We have chosen $f_{k}$ to be real and positive at the initial time $\eta_{i}$, but that means no loss of generality because of global phase freedom. With this change of variables, the mode equation (B3) becomes

$$
W_{k}^{2}=\Omega_{k}^{2}-\frac{1}{2}\left[\frac{\ddot{W}_{k}}{W_{k}}-\frac{3}{2}\left(\frac{\dot{W}_{k}}{W_{k}}\right)^{2}\right] .
$$

Solving this equation iteratively, starting with $W_{k}=\Omega_{k}$, yields an adiabatic expansion for $W_{k}$. This is the WKB solution of Eq. (B8), and the mode associated with it through Eq. (B7) is a WKB mode. The WKB solution can be expanded in inverse powers of $k$ as

$$
W_{k}=k+\frac{M^{2}}{2 k}-\frac{M^{4}+\left(d^{2} M^{2} / d \eta^{2}\right)}{8 k^{3}}+O\left(k^{-5}\right) .
$$

The fourth-order adiabatic approximation to the WKB solution, which contains up to fourth-order derivatives of the scale factor, is obtained by truncating the iterative procedure after the second iteration:

$$
\left(W_{k}^{(4)}\right)^{2}=\Omega_{k}^{2}-\frac{1}{2}\left[\frac{\ddot{\Omega}_{k}}{\Omega_{k}}-\frac{3}{2}\left(\frac{\dot{\Omega}_{k}}{\Omega_{k}}\right)^{2}\right],
$$

and agrees with the exact solution (B9) up to $O\left(k^{-3}\right)$. The bare expectation value (B6) can be renormalized by the socalled adiabatic subtraction procedure [89-92], which amounts to subtracting the same expectation value computed with the fourth-order adiabatic approximation to the WKB modes:

$$
\left\langle T_{\mu}^{\mu}\right\rangle_{\mathrm{ren}}=\left\langle T_{\mu}^{\mu}\right\rangle-\left\langle T_{\mu}^{\mu}\right\rangle^{(4)},
$$

where $\left\langle T_{\mu}^{\mu}\right\rangle^{(4)}$ is the trace (B6) associated with the modes (B7) with $W_{k}$ given by Eq. (B10). This procedure has been shown to be equivalent to covariant regularization and renormalization methods specialized to RobertsonWalker spacetimes [93,94].

In this Appendix we are concerned with the renormalized expectation value of the trace at the initial time $\eta_{i}$. The first question that we want to address is for which homogeneous and isotropic vacua (B11) is finite at the initial time. In other words, we want to determine the class of initial conditions for the modes $\left\{f_{k}\right\}$ that render (B11) finite at $\eta_{i}$. According to Eq. (B7), the most general initial conditions that fulfill the Wronskian condition (B4) are

$$
\begin{gathered}
f_{k}\left(\eta_{i}\right)=\frac{1}{\sqrt{2 w_{k}}}, \\
\dot{f}_{k}\left(\eta_{i}\right)=\left(-i w_{k}+\frac{v_{k}}{2}\right) f_{k}\left(\eta_{i}\right),
\end{gathered}
$$

where $w_{k}$ and $v_{k}$ are two arbitrary real functions of $k$ (with $w_{k}>0$ ). By making use of Eqs. (B6), (B7), and (B11)(B13), the renormalized expectation value of the trace at the initial time can be explicitly written as follows:

$$
\begin{aligned}
\left\langle T_{\mu}^{\mu}\right\rangle_{\mathrm{ren}}\left(\eta_{i}\right)= & -\frac{3 \nu}{2 \pi^{2} a_{i}^{4}} \int_{0}^{\infty} d k k^{2}\left\{\dot{h_{i}}\left[\frac{1}{w_{k}}-\frac{1}{W_{k i}^{(4)}}\right]\right. \\
& +h_{i}\left[\frac{v_{k}}{w_{k}}+\frac{\dot{W}_{k i}^{(4)}}{\left(W_{k i}^{(4)}\right)^{2}}\right]-\left[w_{k}-W_{k i}^{(4)}\right] \\
& \left.+\Omega_{k i}^{2}\left[\frac{1}{w_{k}}-\frac{1}{W_{k i}^{(4)}}\right]-\left[\frac{v_{k}^{2}}{4 w_{k}}-\frac{\left(\dot{W}_{k i}^{(4)}\right)^{2}}{4\left(W_{k i}^{(4)}\right)^{3}}\right]\right\},
\end{aligned}
$$

where the subscript $i$ indicates that the function is eval- 
uated at the initial time $\eta_{i}$. This integral is finite provided that the integrand falls off faster than $k^{-1}$ as $k \rightarrow \infty$, which is achieved if

$$
\begin{aligned}
& \left|w_{k}-W_{k i}\right|<O\left(k^{-3}\right), \\
& \left|v_{k}+\frac{\dot{W}_{k i}}{W_{k i}}\right|<O\left(k^{-2}\right),
\end{aligned}
$$

when $k \rightarrow \infty$. Here we have used the fact that $W_{k}^{(4)}$ and $W_{k}$ agree up to order $O\left(k^{-3}\right)$. Among all possible homogeneous and isotropic vacua this condition selects those which have a finite trace of the renormalized stress-tensor expectation value at the initial time. They correspond to the socalled fourth-order adiabatic vacua.

Let us now concentrate ourselves on the particular class of vacuum states that have been considered in this paper. They were defined by means of an auxiliary scale factor $a_{\Psi}$ in the time domain $\left(-\infty, \eta_{i}\right]$, which gives a timedependent mass $M_{\Psi}^{2}=\nu a_{\Psi}^{2} R_{\Psi}$. Note that $M_{\Psi}$ must vanish at past infinity [see Eq. (7)] and is otherwise an arbitrary function of the conformal time. The vacuum states that we have considered, given by Eq. (6), correspond to the following initial conditions for the modes:

$$
\begin{aligned}
& f_{k}\left(\eta_{i}\right)=f_{k}^{\Psi}\left(\eta_{i}\right), \\
& \dot{f}_{k}\left(\eta_{i}\right)=\dot{f}_{k}^{\Psi}\left(\eta_{i}\right),
\end{aligned}
$$

where $f_{k}^{\Psi}(\eta)$ is the solution of the mode equation (B3) with $M_{\Psi}$ replacing $M$,

$$
\ddot{f}_{k}^{\Psi}+\Omega_{k \Psi}^{2} f_{k}^{\Psi}=0,
$$

and behaves as a standard plane wave at past infinity:

$$
f_{k}^{\Psi}(\eta) \rightarrow \frac{1}{\sqrt{2 k}} e^{-i k \eta} \quad \text { when } \eta \rightarrow-\infty .
$$

In fact, we can be more specific about these initial conditions. In this paper we have computed the expectation value of the trace of the stress tensor to order $\nu^{2}$. Since the trace is of order $\nu$ for any state (except for the trace anomaly terms, which are of order $\nu^{0}$ and independent of the state), we only need to know the modes $\left\{f_{k}\right\}$ to order $\nu$ [see Eq. (B6)]. Solving Eq. (B19) to this order, and choosing appropriately the global phase, we find that our initial conditions can be written as in Eqs. (B12) and (B13) with

$$
\begin{aligned}
& w_{k}=k+\int_{-\infty}^{\eta_{i}} d \eta \sin \left[2 k\left(\eta_{i}-\eta\right)\right] M_{\Psi}^{2}(\eta)+O\left(\nu^{2}\right), \\
& v_{k}=-2 \int_{-\infty}^{\eta_{i}} d \eta \cos \left[2 k\left(\eta_{i}-\eta\right)\right] M_{\Psi}^{2}(\eta)+O\left(\nu^{2}\right) .
\end{aligned}
$$

The second issue that we are concerned with in this Appendix is how the constraints (B15) and (B16) affect our particular class of vacuum states, associated with the initial conditions (B17) and (B18). To answer this question we do not need to use any perturbative approximation like that in Eqs. (B21) and (B22). Indeed, in the ultraviolet limit $k \rightarrow \infty$ Eq. (B19) admits a solution of the form (B7) with a frequency $W_{k}^{\Psi}$ given by Eq. (B9) with $M_{\Psi}$ replacing $M$. Let us call it $\tilde{f}_{k}^{\Psi}$. Since $M_{\Psi}$ vanishes at past infinity, this solution behaves as a standard plane wave at past infinity, i.e., it satisfies Eq. (B20). Hence, $f_{k}^{\Psi}(\eta)=\tilde{f}_{k}^{\Psi}(\eta)$ in the ultraviolet limit. The initial conditions (B17) and (B18) can then be written as in Eqs. (B12) and (B13) with $w_{k}$ and $v_{k}$ given by

$$
\begin{gathered}
w_{k}=W_{k i}^{\Psi}, \\
v_{k}=-\frac{\dot{W}_{k i}^{\Psi}}{W_{k i}^{\Psi}}
\end{gathered}
$$

for $k \rightarrow \infty$, where the subscript $i$ again means that the function is evaluated at the initial time $\eta_{i}$. The conditions (B15) and (B16), which select the fourth-order adiabatic vacua, become

$$
\begin{gathered}
\left|W_{k i}^{\Psi}-W_{k i}\right|<O\left(k^{-3}\right), \\
\left|-\frac{\dot{W}_{k i}^{\Psi}}{W_{k i}^{\Psi}}+\frac{\dot{W}_{k i}}{W_{k i}}\right|<O\left(k^{-2}\right) .
\end{gathered}
$$

Making use of the expansion in Eq. (B9), we can rewrite these inequalities as

$$
\begin{aligned}
& \mid \frac{1}{2 k}\left[M_{\Psi i}^{2}-M_{i}^{2}\right]-\frac{1}{8 k^{3}}\left[M_{\Psi i}^{4}-M_{i}^{4}+\left(d^{2} M_{\Psi}^{2} / d \eta^{2}\right)_{i}\right. \\
& \left.\quad-\left(d^{2} M^{2} / d \eta^{2}\right)_{i}\right]+O\left(k^{-5}\right) \mid<O\left(k^{-3}\right), \\
& \left|\frac{1}{2 k^{2}}\left[\left(d M_{\Psi}^{2} / d \eta\right)_{i}-\left(d M^{2} / d \eta\right)_{i}\right]+O\left(k^{-4}\right)\right|<O\left(k^{-2}\right),
\end{aligned}
$$

which are satisfied if and only if

$$
\begin{aligned}
M_{\Psi i}^{2}= & M_{i}^{2}, \quad\left(d M_{\Psi}^{2} / d \eta\right)_{i}=\left(d M^{2} / d \eta\right)_{i}, \\
& \left(d^{2} M_{\Psi}^{2} / d \eta^{2}\right)_{i}=\left(d^{2} M^{2} / d \eta^{2}\right)_{i} .
\end{aligned}
$$

These are precisely the conditions (40), which we found by other means in Sec. IV B. Therefore, we conclude that requiring the scale factors before and after the initial time to match smoothly enough, according to Eq. (40), is equivalent to demanding that the initial vacuum state is fourth-order adiabatic. 
[1] A. H. Guth, Phys. Rev. D 23, 347 (1981).

[2] A. D. Linde, Phys. Lett. 108B, 389 (1982).

[3] A. Albrecht and P. J. Steinhardt, Phys. Rev. Lett. 48, 1220 (1982).

[4] A. D. Linde, Phys. Lett. 129B, 177 (1983).

[5] A. D. Linde, Particle Physics and Inflationary Cosmology (Harwood Academic, Amsterdam, 1990).

[6] G. F. Smoot et al., Astrophys. J. 396, L1 (1992).

[7] C. L. Bennett et al., Astrophys. J. Suppl. Ser. 148, 1 (2003).

[8] H. V. Peiris et al., Astrophys. J. Suppl. Ser. 148, 213 (2003).

[9] D. N. Spergel et al., Astrophys. J. Suppl. Ser. 170, 377 (2007).

[10] S. Perlmutter et al., Astrophys. J. 517, 565 (1999).

[11] A. G. Riess et al., Astron. J. 116, 1009 (1998).

[12] U. Seljak, A. Slosar, and P. McDonald, J. Cosmol. Astropart. Phys. 10 (2006) 014.

[13] M. Tegmark et al., Phys. Rev. D 74, 123507 (2006).

[14] T. Giannantonio et al., Phys. Rev. D 74, 063520 (2006).

[15] D. J. Eisenstein, Astrophys. J. 633, 560 (2005).

[16] N. C. Tsamis and R.P. Woodard, Nucl. Phys. B474, 235 (1996).

[17] N. C. Tsamis and R. P. Woodard, Ann. Phys. (N.Y.) 253, 1 (1997).

[18] V.F. Mukhanov, L.R.W. Abramo, and R.H. Brandenberger, Phys. Rev. Lett. 78, 1624 (1997).

[19] L. R.W. Abramo, R.H. Brandenberger, and V.F. Mukhanov, Phys. Rev. D 56, 3248 (1997).

[20] L. R. W. Abramo and R. P. Woodard, Phys. Rev. D 60, 044010 (1999).

[21] B. Losic and W. G. Unruh, Phys. Rev. D 72, 123510 (2005).

[22] S. B. Giddings, D. Marolf, and J. B. Hartle, Phys. Rev. D 74, 064018 (2006).

[23] L. R. W. Abramo and R. P. Woodard, Phys. Rev. D 65, 063515 (2002).

[24] G. Geshnizjani and R. Brandenberger, Phys. Rev. D 66, 123507 (2002).

[25] J. Garriga and T. Tanaka, Phys. Rev. D 77, 024021 (2008).

[26] N. C. Tsamis and R. P. Woodard, arXiv:0708.2004v2.

[27] D. Espriu, T. Multamäki, and E. C. Vagenas, Phys. Lett. B 628, 197 (2005).

[28] J. F. Donoghue, Phys. Rev. Lett. 72, 2996 (1994).

[29] J. F. Donoghue, Phys. Rev. D 50, 3874 (1994).

[30] N. E. J. Bjerrum-Bohr, J. F. Donoghue, and B. R. Holstein, Phys. Rev. D 68, 084005 (2003); 71, 069904(E) (2005).

[31] N.D. Birrell and P.C.W. Davies, Quantum Fields in Curved Space (Cambridge University Press, Cambridge, England, 1994).

[32] R. M. Wald, Quantum Field Theory in Curved Spacetime and Black Hole Thermodynamics (The University of Chicago Press, Chicago, 1994).

[33] E. E. Flanagan and R. M. Wald, Phys. Rev. D 54, 6233 (1996).

[34] T. S. Bunch and P. C. W. Davies, Proc. R. Soc. A 360, 117 (1978).

[35] J. S. Dowker and R. Critchley, Phys. Rev. D 13, 3224 (1976).

[36] A. A. Starobinsky, Phys. Lett. 91B, 99 (1980).

[37] S. Wada and T. Azuma, Phys. Lett. 132B, 313 (1983).
[38] D. Lopez Nacir and F. D. Mazzitelli, Phys. Rev. D 76, 024013 (2007).

[39] P. R. Anderson, W. Eaker, S. Habib, C. Molina-París, and E. Mottola, Phys. Rev. D 62, 124019 (2000).

[40] S. Habib, C. Molina-París, and E. Mottola, Phys. Rev. D 61, 024010 (1999).

[41] C. W. Misner, K. S. Thorne, and J. A. Wheeler, Gravitation (Freeman, San Francisco, 1973).

[42] L. Parker, in Recent Developments in Gravitation, edited by S. Deser and M. Levy (Plenum Press, New York, 1979).

[43] A. Campos and E. Verdaguer, Phys. Rev. D 49, 1861 (1994).

[44] A. Campos and E. Verdaguer, Int. J. Theor. Phys. 36, 2525 (1997).

[45] E. Calzetta, A. Campos, and E. Verdaguer, Phys. Rev. D 56, 2163 (1997).

[46] G. Leibbrandt, Rev. Mod. Phys. 47, 849 (1975).

[47] R. Martín and E. Verdaguer, Phys. Lett. B 465, 113 (1999).

[48] R. Martín and E. Verdaguer, Phys. Rev. D 60, 084008 (1999).

[49] F. C. Lombardo and F. D. Mazzitelli, Phys. Rev. D 55, 3889 (1997).

[50] B. L. Hu and E. Verdaguer, Classical Quantum Gravity 20, R1 (2003).

[51] B. L. Hu and E. Verdaguer, Living Rev. Relativity 7, 3 (2004).

[52] E. Calzetta and B. L. Hu, Phys. Rev. D 49, 6636 (1994).

[53] B. L. Hu and A. Matacz, Phys. Rev. D 51, 1577 (1995).

[54] B. L. Hu and S. Sinha, Phys. Rev. D 51, 1587 (1995).

[55] A. Campos and E. Verdaguer, Phys. Rev. D 53, 1927 (1996).

[56] A. Roura and E. Verdaguer, Phys. Rev. D 60, 107503 (1999).

[57] L. Schwartz, Théory des Distributions (Hermann, Paris, 1966).

[58] G. T. Horowitz, Phys. Rev. D 21, 1445 (1980).

[59] F. Cooper and E. Mottola, Phys. Rev. D 40, 456 (1989).

[60] J. Z. Simon, Phys. Rev. D 41, 3720 (1990).

[61] J. Z. Simon, Phys. Rev. D 43, 3308 (1991).

[62] J.Z. Simon, Phys. Rev. D 45, 1953 (1992).

[63] L. Parker and J.Z. Simon, Phys. Rev. D 47, 1339 (1993).

[64] B. L. Hu and A. Roura, Phys. Rev. D 76, 124018 (2007).

[65] V.P. Frolov and A. I. Zelnikov, Phys. Rev. D 29, 1057 (1984).

[66] E. E. Flanagan, Classical Quantum Gravity 21, 3817 (2004).

[67] T. Futamase, T. Rothman, and R. Matzner, Phys. Rev. D 39, 405 (1989).

[68] G. Pérez-Nadal, A. Roura, and E. Verdaguer, arXiv:0806.2634.

[69] J. A. Cabrer and D. Espriu, arXiv:0710.0855.

[70] P. R. Anderson, C. Molina-París, and E. Mottola, Phys. Rev. D 67, 024026 (2003).

[71] P. R. Anderson, C. Molina-París, and E. Mottola (unpublished).

[72] J. F. Donoghue, in The Eighth Marcel Grossmann Meeting, edited by T. Piran and R. Ruffini (World Scientific, Singapore, 1999).

[73] C. P. Burgess, Living Rev. Relativity 7, 5 (2004).

[74] S. Weinberg, Phys. Rev. D 72, 043514 (2005).

[75] S. Weinberg, Phys. Rev. D 74, 023508 (2006). 
[76] B. L. Hu, A. Roura, and E. Verdaguer, Phys. Rev. D 70, 044002 (2004).

[77] A. Roura and E. Verdaguer, Int. J. Theor. Phys. 38, 3123 (1999).

[78] B. Losic and W. G. Unruh, Phys. Rev. D 74, 023511 (2006).

[79] J. Schwinger, J. Math. Phys. (N.Y.) 2, 407 (1961).

[80] L. V. Keldysh, Sov. Phys. JETP 20, 1018 (1965) [Zh. Eksp. Teor. Fiz. 47, 1515 (1964)].

[81] K. Chou, Z. Su, B. Hao, and L. Yu, Phys. Rep. 118, 1 (1985).

[82] R. D. Jordan, Phys. Rev. D 33, 444 (1986).

[83] E. Calzetta and B.L. Hu, Phys. Rev. D 35, 495 (1987).

[84] R. P. Feynman and F. L. Vernon, Ann. Phys. (N.Y.) 24, 118 (1963).
[85] R. P. Feynman and A. R. Hibbs, Quantum Mechanics and Path Integrals (McGraw-Hill, New York, 1965).

[86] J. Garriga, O. Pujolàs, and T. Tanaka, Nucl. Phys. B605, 192 (2001).

[87] J. Garriga, O. Pujolàs, and T. Tanaka, Nucl. Phys. B655, 127 (2003).

[88] J. Lindig, Phys. Rev. D 59, 064011 (1999).

[89] S. A. Fulling and L. Parker, Ann. Phys. (N.Y.) 87, 176 (1974).

[90] L. Parker and S. A. Fulling, Phys. Rev. D 9, 341 (1974).

[91] T. S. Bunch, J. Phys. A 13, 1297 (1980).

[92] F. D. Mazzitelli, J.P. Paz, and M. A. Castagnino, Phys. Rev. D 36, 2994 (1987).

[93] N. D. Birrell, Proc. R. Soc. B 361, 513 (1978).

[94] P. R. Anderson and L. Parker, Phys. Rev. D 36, 2963 (1987). 